\title{
EXISTENCE OF EQUILIBRIUM IN SINGLE AND DOUBLE PRIVATE VALUE AUCTIONS ${ }^{1}$
}

\begin{abstract}
By MATTHEW O. JACKSON AND JEROEN M. SwinKeLS ${ }^{2}$
We show existence of equilibria in distributional strategies for a wide class of private value auctions, including the first general existence result for double auctions. The set of equilibria is invariant to the tie-breaking rule. The model incorporates multiple unit demands, all standard pricing rules, reserve prices, entry costs, and stochastic demand and supply. Valuations can be correlated and asymmetrically distributed. For double auctions, we show further that at least one equilibrium involves a positive volume of trade. The existence proof establishes new connections among existence techniques for discontinuous Bayesian games.
\end{abstract}

KEYWORDS: Auctions, double auctions, equilibrium, existence, invariance, private values.

\section{INTRODUCTION}

AUCTIONS ARE GENERALLY modeled by allowing players to choose from continuum bidding spaces. However, players' payoffs in auctions are discontinuous at points of tied bids, which in the face of continuum bidding spaces makes existence of equilibrium difficult to prove. Much of what is known about existence of equilibrium in auctions comes from exhibiting equilibrium strategies in symmetric settings (for instance in Milgrom and Weber (1982)) or relying on monotonicity arguments (e.g., Athey (2001) or Maskin and Riley (2000)). This leaves open the question of existence of equilibria in many auction settings, such as those where distributions fail to satisfy nice monotonicity properties, and settings including the important class of double auctions.

Since the fundamental difficulty in these proofs revolves around the continuity of the bid space, one might well ask why one bothers to impose such an assumption. After all, one could argue that all true bid spaces are in fact discrete. However, continuum bid spaces are a very useful approximation as they simplify the analysis, allowing one, for example, to use calculus to characterize equilibria. Thus, almost all models of auctions use continuum bid spaces and so it is important to understand when equilibria exist in such models. Moreover, discrete bid spaces can introduce some pathological equilibria. For example, both Jackson (1999) and Jackson, Simon, Swinkels, and Zame (2002)

\footnotetext{
${ }^{1}$ This paper supersedes the second part of Jackson and Swinkels (1999). That paper was split: The results on existence of equilibria in Bayesian games with type-dependent sharing were combined with Simon and Zame (1999) to become Jackson, Simon, Swinkels, and Zame (2002). The results on existence of equilibria in a class of private value auctions have evolved into this paper.

${ }^{2}$ We thank Leo Simon, Bill Zame, Mark Satterthwaite, and Phil Reny for helpful conversations. We also thank Kim Border, Martin Cripps, John Nachbar, Larry Samuelson, Tianxiang Ye, three anonymous referees, and the editor for helpful comments and suggestions. Financial support from the National Science Foundation under Grant SES-9986190 and from the Boeing Center for Technology and Information Management is gratefully acknowledged.
} 
(henceforth JSSZ) show examples where the game with finite bid spaces has an equilibrium, while the continuum game does not. In each, one positive measure set of player types concentrates on one bid, while another concentrates on the next available bid. This does not strike us as what we had in mind when discussing equilibrium. Existence of equilibrium with continuum bidding spaces helps to establish the existence of a nonpathological equilibrium in the discrete versions of these auctions. ${ }^{3,4}$

This paper has three main results about equilibria in auctions: existence, invariance, and nontriviality. By existence, of course we mean that the set of equilibria is nonempty. By invariance, we mean that the set of equilibria of auctions in our class does not depend on the precise tie-breaking rule. Invariance actually plays a key role in our existence proof. And, by nontriviality, we mean that in the set of equilibria there is always one in which trade occurs with positive probability.

Our existence and invariance results apply to auctions that can be single or double sided, including settings where players are unsure at the time that they bid whether they will be net buyers or net sellers. The pricing rule can be quite general, including both the uniform and discriminatory cases, all pay features, entry costs, reserve prices, and many other variations. Demands and supplies may be for multiple units, and valuations can be asymmetrically distributed and follow very general correlation patterns; neither independence nor any form of affiliation is assumed. Finally, in addition to covering standard tie-breaking rules, we also cover more general tie-breaking rules, including allowing the auctioneer to use information in breaking ties that he is typically not assumed to have, such as the true values of the players.

In double auctions, showing that an equilibrium exists does not close the issue. The difficulty is that double auctions have a degenerate equilibrium in which all buyers bid 0 , and all sellers bid $\bar{v}$, the upper bound of values. ${ }^{5}$ Our second main result establishes existence of a nondegenerate equilibrium for each auction in our class; that is, one where trade occurs with positive probability. So, our results are not vacuous in settings where there exist degenerate

\footnotetext{
${ }^{3} \mathrm{JSSZ}$ also argue that one should be uncomfortable with the equilibria of discrete bid auctions if one does not know that they correspond to analogs in the continuum, because absent an existence result for the continuum case, there is no assurance that the equilibrium does not depend on the particular discretization chosen. For example, it might be critical whether different players' available bids overlap or are disjoint.

${ }^{4}$ The underlying existence theorems used (either JSSZ or Reny (1999)) include an upper hemicontinuity result. Hence, the fact (which we establish) that all equilibria of the continuum game are tie-free implies the existence of almost tie-free equilibria in games with small bid increments.

${ }^{5}$ Such no-trade equilibria are notoriously difficult to overcome in some other settings. For instance, see the discussion of positive trade equilibria in market games in Dubey and Shubik (1978) and Peck, Shell, and Spear (1992).
} 
no-trade equilibria. Taken together, these two results provide the first general existence result of any form for the double auction setting. ${ }^{6}$

Our results, and the relation between invariance and existence, can be understood as follows.

First, let us discuss invariance. Let an omniscient tie-breaking rule be a map from bids and values to distributions over allocations that respects the order of bids. Such maps include standard tie-breaking rules where ties are broken by some simple randomization or fixed priorities, but also include weirder rules, such as ones where the auctioneer uses other information such as players' types and, for instance, breaks ties efficiently. Say that a tie-breaking rule is trademaximizing if ties between buyers and sellers are broken in favor of buyers. Then, the invariance result states that if a strategy profile forms an equilibrium for one omniscient tie-breaking rule, it remains an equilibrium for any other trade-maximizing omniscient tie-breaking rule. Two ideas underlie this result. First, regardless of the tie-breaking rule, given the continuous distribution over types, players will not want to play in such a way as to be involved in ties with positive probability (except that a buyer and a seller may be tied provided trade occurs between them), as otherwise some player would benefit from raising or lowering his bids contingent on some types. So, changing to some other trade-maximizing tie-breaking rule does not change payoffs under the original strategy profile. Second, if a player has an improving deviation relative to some strategy profile and tie-breaking rule, then there is a slight modification of the deviation that is still improving and also avoids ties. But then, any improving deviation at one tie-breaking rule implies an improving deviation under any other tie-breaking rule as well. Hence, replacing one tie-breaking rule with another does not change the best replies of the players at the equilibrium. ${ }^{7}$

With the invariance result in hand, there are at least three ways to complete the proof of existence. First, one can use the main result of JSSZ. They prove existence of equilibria in Bayesian games in which tie-breaking is allowed to be "endogenous" in the sense that it is determined as part of the equilibrium and can depend (in an incentive compatible way) on the private information of players. A fortiori this is an equilibrium with omniscient tie-breaking, and hence, by the invariance result the strategies involved are also an equilibrium for any trade-maximizing tie-breaking rule including any standard one.

The invariance result can also be used to establish existence via the theorem of Reny (1999) (henceforth Reny). We know of two such approaches. In one approach, one uses an auxiliary result of Reny's (Proposition 3.2), involving

\footnotetext{
${ }^{6}$ Williams (1991) shows existence in the particular case that the price is determined by the lowest winning buy bid. This case is easier, because (a) the sellers then have as a weakly dominant strategy to bid their values and hence (b) the game can be thought of as a one-sided auction with a hidden reserve price schedule, for which degenerate equilibria are not an issue.

${ }^{7} \mathrm{An}$ important precursor to this idea of substitution of tie-breaking rules can be found in Lebrun (1996).
} 
a condition called reciprocal upper semicontinuity (due to Simon (1987), who generalizes Dasgupta and Maskin (1986)). This requires that if one player's payoff jumps down at a discontinuity, some other player's payoff jumps up. Under standard tie-breaking, this condition is not satisfied (see footnote 13 of Reny). However, if tie-breaking is chosen to maximize the sum of player payoffs, then reciprocal upper semicontinuity is satisfied (as is the stronger condition of Dasgupta and Maskin). Since such tie-breaking is among the omniscient tie-breaking rules, this (coupled with invariance) again implies the result.

We use a different approach in applying Reny's result, working directly with his main theorem. We think this approach illustrates an important way of applying Reny's result more generally, and that it hints at a deeper connection between Reny and JSSZ. Reny's basic condition, better-reply-security, requires checking that there are paying deviations, not just relative to payoffs actually available at non-Nash strategy profiles, but also relative to all payoffs that can be generated as a limit of nearby strategy profiles. The deviation must pay, not only relative to the original strategy profile, but to small perturbations. The first part of applying Reny thus involves the potentially daunting task of characterizing the set of payoff profiles available from such limits at any given point, which in particular can be quite complicated at points of discontinuity such as ties. We show, however, that any such payoff profile can be induced by an appropriately chosen omniscient tie-breaking rule. Then, using the first idea underlying our invariance result, that players will play so as to avoid being involved in ties regardless of the tie-breaking rule, establishes the desired condition. We find it illuminating that all three routes to existence rely on some form of omniscient tie-breaking. ${ }^{8}$

The last piece of the puzzle is to show that there are nondegenerate (positive trade) equilibria in settings such as the double auction. Our approach is to "seed" the auction with a nonstrategic player who is present with small probability, and who then makes buy or sell bids uniform over the range of values. With this extra player present, it no longer makes sense for buyers to always offer 0 , or sellers to always ask $\bar{v}$, since such bids never trade, while one could bid more generously, and sometimes profitably trade with the nonstrategic player. What is less clear is that this implies an amount of trade that does not vanish as the probability of the nonstrategic player being present goes to 0 . The key is to show that once the nonstrategic player is present, competition to trade with him will push the bids of buyers with high values well above 0 and sellers with low values well below $\bar{v}$. Essentially then, the extra player sets off a cascade, resulting in a positive amount of trade even as the probability that the extra player is present vanishes.

Section 2 discusses related literature. Section 3 presents our private value auction setting. Section 4.1 shows that the equilibria in such auctions are invariant to the tie-breaking rule. In Section 4.2, we show how invariance implies

${ }^{8}$ This is also true of Lebrun (1996) and Maskin and Riley (2000). 
existence of equilibria for the auctions in our class. Section 5 shows that these equilibria can be chosen to have nondegenerate trade in auction environments where no-trade equilibria are a possibility, such as double auctions. An Appendix contains the proofs.

\section{RELATED LITERATURE}

The method of proof we use for our first result, demonstrating existence with a nonstandard tie-breaking rule and then showing that the tie-breaking rule can be changed to a standard one, is reminiscent of Maskin and Riley (2000). ${ }^{9}$ The strategy of Maskin and Riley's proof is to consider a sequence of auctions with finite bid spaces, in which, in the event of a tie, a Vickrey auction takes place. For the auctions they consider, the Vickrey auction is enough to guarantee that payoffs are preserved in the limit as the finite grid of bids grows fine. They then argue that these ties would never occur in equilibrium anyway, and so the equilibrium is in fact a standard one. So, like them, we show existence in a game where one does something strange in the event of a tie and then work backward to show that in many cases of interest this was irrelevant, in our case, by applying our invariance result. Within the class of private value auctions, we cover a substantially broader set of cases than those of Maskin and Riley, essentially because our tie-breaking methods allow for potentially stranger rules. This allows us to handle equilibrium where bidding is not monotone in type, and thus to cover a wide variety of auction formats and information structures not handled in the previous literature. On the other hand, Maskin and Riley's results hold for some nonprivate value auctions (with affiliated types) while our result does not. It is an open question how far techniques like those in this paper extend beyond private value auctions.

As discussed above, the idea of getting existence in games with augmented message spaces is also related to Lebrun (1996), who looks at first price single unit private value auctions in which bids are augmented by messages that turn out to be irrelevant. He notes that in augmenting the game, and letting tiebreaking depend on the message sent, he is doing something reminiscent of what Simon and Zame (1990) do in games of complete information. ${ }^{10}$ Thus, in the specific setting he studies, Lebrun's technique parallels the one we use here.

This paper is also related to the literature on existence in games with continuum type spaces, including, for example, Dasgupta and Maskin (1986),

\footnotetext{
${ }^{9}$ This technique is also used by JSSZ who argue that if valuations are private and the distribution is atomless and satisfies some other continuity conditions, then there exists an equilibrium in a first price auction with standard tie-breaking (see their Example 3).

${ }^{10}$ The idea of using messages to restore limit continuity in a finite approximation setting should also be credited to Manelli (1996), who uses such messages to restore equilibria in infinite signaling games.
} 
Simon (1987), and of course Reny (1999). Reny shows that his condition applies in a multiple unit, private value, pay your bids auction, a case for which we also prove existence (see his Example 5.2). A recent working paper by Bresky (1999) uses a different line of attack to apply Reny's result to private value auctions. Neither paper covers the class of settings covered here.

Of course, none of the previous literature has anything to say about the problem of no-trade equilibria in double auctions.

Thus we move beyond the previous literature in four ways:

(i) We show a broad invariance property across tie-breaking rules for the equilibria of private value auctions.

(ii) We show that the invariance property and consideration of nonstandard tie-breaking allows for the straightforward application of either JSSZ or Reny. The way in which these results can be leveraged should be interesting in its own right and potentially of wider applicability.

(iii) This provides an existence result for private value auctions that covers substantially more ground than previous results, even in the single auction case.

(iv) Finally, we show there always exists an equilibrium in which there is a positive probability of trade. This overcomes the problem of no-trade equilibria, and provides the first existence result of any form for double auctions. ${ }^{11}$

A number of papers approach the existence question in single unit auctions by examining the associated set of differential equations. A strength of this approach as compared to ours is that it allows for interesting comparative static and uniqueness results. Such an approach requires much more structure regarding the distributions of valuations than we require here. For some leading examples, see Milgrom and Weber (1982), Lebrun (1999), Bajari (1996), and Lizzeri and Persico (2000).

Athey (2001) considers conditions on games such that a monotone comparative statics result applies to the best bid of a player as his signal varies. Essentially, one imposes a condition under which, if all of $i$ 's opponents are using an increasing strategy, then $i$ has a best response in increasing strategies. A strength of Athey's result is that it does not rest on private values. It does however, require a single-dimensional type space with something akin to the monotone likelihood ratio property (MLRP; see Example 1). Recent work by McAdams (2003) and Kazumori (2003) extends this to a multiple-dimensional setting with independent types, in the former case with a discrete bid space, while in the later, with a continuum.

Each of the auction papers mentioned above derives the existence of pure strategy equilibria, while in general we show only the existence of equilibria

\footnotetext{
${ }^{11}$ Since the first writing of this paper (1999), others have also looked at existence of equilibrium in double auctions. Fudenberg, Mobius, and Szeidl (2003) show existence of equilibrium in double auctions with sufficiently many players. Perry and Reny (2003) address existence in double auctions with a discrete bid space. These papers all work in a symmetric affiliated or conditionally independent setting, and derive increasing equilibria. Our setting has neither symmetry nor affiliation, but does not rule out that the equilibria found involve mixing.
} 
in distributional strategies. This is partly due to the methods we employ, but mostly due to the broadness of the class of distributions of valuations that we admit. In particular (see Example 2) not all auctions in our setting have such pure strategy equilibria, and so a result covering these auctions can at most claim existence of mixed strategy equilibria. In some settings with positively related valuations, one can start from our existence result and then independently deduce that all equilibria must be in increasing (and therefore essentially pure) strategies. We present one such result, generalizing McAdams (2003) and Kazumori (2003) for the case of private values. ${ }^{12}$ See Reny and Zamir (2003) and Krishna (2003) for other interesting recent work on pure strategy equilibria.

\section{THE SETTING AND A CLASS OF AUCTIONS}

We begin by presenting our model of private value auctions. The model treats single and double auctions (as well as hybrids) in a single framework.

\subsection{The Setting}

Let us first describe the setting in terms of the players, objects, valuations, and uncertainty.

PlAYERS: There are players $N=\{1, \ldots, n\}$, along with a nonstrategic "player" 0 , who can act as the seller, for example, in a single sided auction.

OBJECTS AND ENDOWMENTS: There is $\ell<\infty$ such that each player $i \in$ $N \cup\{0\}$ has an endowment of $e_{i} \in\{0,1, \ldots, \ell\}$ indivisible objects. Objects are identical. Let $e=\left(e_{0}, e_{1}, \ldots, e_{n}\right)$ denote the vector of endowments.

VALUATIONS: Each player $i \in N$ desires at most $\ell$ objects. Player $i$ 's valuations are represented by $v_{i}=\left(v_{i 1}, \ldots, v_{i \ell}\right)$. The interpretation is that $i$ has marginal value $v_{i h}$ for an $h$ th object.

ASSUMPTION 1 (Private Values): Player i receives value $\sum_{h=1}^{H} v_{i h}$ from having $H$ objects.

For $h \leq e_{i}$, we say that $v_{i h}$ is a sell value. For $h>e_{i}$, we say that $v_{i h}$ is a buy value.

Let $v=\left(v_{1}, \ldots, v_{n}\right)$ be the vector of valuations of the players.

\footnotetext{
${ }^{12}$ With independent types, these papers can deal with interdependent values, which we do not. We would like to be clear that while our basic existence results for equilibria in distributional strategies (including nontrivial equilibria in double auctions) predate McAdams and Kazumori's, our corollary on pure strategy equilibria postdates theirs, and, indeed is partly motivated by their work.
} 
TYPES: We say that $\theta_{i}=\left(e_{i}, v_{i}\right)$ is the type of player $i$, and let $\theta=(e, v)$ denote the vector of types of all players. Let $\Theta_{i} \subset\{0,1, \ldots, \ell\} \times \mathbb{R}^{\ell}$ be the space of possible types for player $i{ }^{13}$ Let $\Theta=\Theta_{0} \times \cdots \times \Theta_{n}$ be the space of type vectors.

ASSUMPTION 2 (Compact Type Space): $\Theta$ is compact.

Let $\bar{v}$ be such that $\Theta \subset\{0,1, \ldots, \ell\}^{n+1} \times[-\bar{v}, \bar{v}]^{(n+1) \ell}$.

UNCERTAINTY: The vector $\theta \in \Theta$ is drawn according to a (Borel) probability measure $P$ on $\Theta$. The marginal of $P$ on $\theta_{i}$ is denoted $P_{i}, i \in\{0, \ldots, n\}$. Without loss of generality, take $\Theta_{i}$ to be the support of $P_{i}$.

AsSUMPTION 3 (Imperfect Correlation): $P$ is absolutely continuous with respect to $\prod_{i=0}^{n} P_{i}$, with continuous Radon-Nikodym derivative $f$.

Assumption 3 puts no restriction on how $e_{i}$ and $v_{i}$ are related, or on the relationship between any two values $v_{i h}$ and $v_{i h^{\prime}}$ for any given player. It simply imposes that $\left(e_{i}, v_{i}\right)$ and $\left(e_{j}, v_{j}\right)$ are not too dependent. For instance, in a twoplayer, one object auction, if $P$ were uniform on the diagonal $\left\{v \mid v_{11}=v_{21}\right\}$, then $v_{1}$ and $v_{2}$ would be perfectly correlated and $P$ would not be absolutely continuous with respect to $P_{1} \times P_{2}$ (the uniform distribution on $[0,1]^{2}$ ). On the other hand, under Assumption 3 types can be "almost perfectly correlated" in the sense that $P$ can place probability one on some small neighborhood of the diagonal.

Assumption 4 (Atomless Distributions): $P_{i}\left(\left\{v_{i h}=x\right\}\right)=0$ for all $i \in N$, $h \in\{1, \ldots, \ell\}$, and $x \in[-\bar{v}, \bar{v}]$.

This assumption rules out that particular values occur with positive probability. It is stronger than just assuming that $P_{i}$ is atomless as it rules out, for example, that $v_{i 1} \equiv 1$ while $v_{i 2}$ is distributed uniformly on $[0,1]$. It allows, however, $v_{i h}=v_{i h^{\prime}}$ with probability one.

We emphasize that we have not imposed any sort of affiliation among different players' values and so the following example is within our setting. Because this auction does not have an equilibrium in nondecreasing strategies, it is not covered by any previous paper on existence in auctions.

EXAMPLE 1: Consider a two player, private value, first price auction. Values are uniformly distributed over the triangle

$$
\left\{\left(v_{1}, v_{2}\right) \mid v_{1} \geq 0, v_{2} \geq 0,1 \geq v_{1}+v_{2}\right\} .
$$

\footnotetext{
${ }^{13}$ We will generally ignore the valuations of the nonstrategic " 0 " player.
} 
Here, higher values of $v_{1}$ correspond to lower expectations of $v_{2}$, and vice versa. This auction has no nondecreasing pure strategy Nash equilibrium. To see why not, suppose to the contrary that such an equilibrium $b_{1}(\cdot), b_{2}(\cdot)$ exists. Let us first argue that $v_{2} \geq b_{2}\left(v_{2}\right)$ for all $v_{2} \in[0,1)$. Suppose not, so that $b_{2}\left(v_{2}^{\prime}\right)>v_{2}^{\prime}$ for some $v_{2}^{\prime} \in[0,1)$. Then, since $b_{2}$ is nondecreasing, $b_{2}\left(v_{2}\right)>v_{2}$ for all $v_{2} \in\left(v_{2}^{\prime}, b_{2}\left(v_{2}^{\prime}\right)\right)$. But, then, since $P\left(v \mid v_{1}<v_{2}, v_{2} \in\left(v_{2}^{\prime}, b_{2}\left(v_{2}^{\prime}\right)\right)\right)>0$, it must be that there is a positive probability that at least one of the players wins with a bid above value ${ }^{14}$ and so would do strictly better to lower his bid to value. This contradicts equilibrium. Now consider a bid by bidder $i$ when his value is above $1-\varepsilon$. He knows that the other bidder's value is below $\varepsilon$, and thus so are the other bidder's bids. Thus, $i$ 's bids should be no more than $\varepsilon$, and so $i$ 's bids for values near 1 are near 0 . Therefore, the only possible equilibrium in nondecreasing strategies is $b_{1}\left(v_{1}\right)=b_{2}\left(v_{2}\right)=0$ for all $\left(v_{1}, v_{2}\right)$, which is clearly not an equilibrium.

ASSUMPTION 5 (Nonincreasing Marginal Valuations):

$$
P\left(\left\{(e, v) \mid v_{i h} \geq v_{i, h+1} \forall i, h\right\}\right)=1 .
$$

Assumption 5 simply states that each player's marginal valuations for objects are nonincreasing in the number of objects. This makes our life easier in terms of keeping track of incentives. In particular, with increasing marginal valuations, a player might find himself submitting the same first and second bids, and simultaneously wishing he could lower his first bid because he dislikes winning one object, but raise his second bid because he likes winning two objects. A related and fuller discussion follows Assumption 9. Whether equilibria still exist in such situations is an open question.

\subsection{The Class of Auctions}

We consider auctions where each player submits a vector of bids, one for each potential object that they may buy or sell. Of course, the auction mechanism may ignore some of this information, but we allow for the possibility that it is used.

\section{Bidding and reserve prices}

For each $i \in\{0, \ldots, n\}$, a bid $b_{i} \in \mathbb{R}^{\ell}$ is a nonincreasing vector of $\ell$ numbers. We assume that there exist real numbers $\underline{b}<\bar{b}$ such that the set of allowable bid vectors for $i$ is the set of nonincreasing vectors

$$
\mathcal{B}_{i} \subset[\underline{b}, \bar{b}]^{\ell} .
$$

\footnotetext{
${ }^{14}$ Either player 2 wins for at least some values of $v_{2} \in\left(v_{2}^{\prime}, b_{2}\left(v_{2}^{\prime}\right)\right)$ or else player 1 must be outbidding 2 even when $v_{1}$ is lower than $v_{2}$.
} 
Let $\mathcal{B}$ denote the set of admissible bid vectors, $\mathcal{B}=\mathcal{B}_{0} \times \mathcal{B}_{1} \times \cdots \times \mathcal{B}_{n}$.

The requirement that bid vectors be nonincreasing is simply a labeling statement, as bids can always be reordered in this manner. It will be consistent with how auctions process bids, in the sense that higher bids are given priority.

For most sensible auctions, the spaces $\mathcal{B}_{i}$ include the compact support of the $v_{i}$ 's and bids outside of that range are weakly dominated. For example, in discriminatory as well as uniform price auctions, a bid $b_{i h}$ above $v_{i h}$ is weakly dominated by a bid at $v_{i h}$. In an all pay auction, a bid above $v_{i h}$ is weakly dominated by bidding 0 . In these settings, one is making no extra restriction on bidder's behavior in imposing the existence of $\bar{b} \cdot{ }^{15}$ In settings where there is a highest sensible bid for a buyer, allowing sell bids above that amount is a convenient way to let a seller "sit out" of the auction, and similarly when there is a lowest sensible bid for sellers.

\section{Timing}

The nonstrategic player moves first, and the remaining players then move simultaneously. So, at time $0, b_{0}$ is announced, then at time 1 each player $i \in N$ observes $\theta_{i}$ and submits a bid $b_{i}{ }^{16,17,18}$

\section{Payments}

The payment that a player makes or receives depends on the number of objects bought or sold. We require that conditional on the number of units that $i$ is allocated, and conditional on the endowment vector, his payment varies

\footnotetext{
${ }^{15}$ For an example in which optimal buy bids may not be bounded from above, consider a third price auction with three players. Suppose that player 3 happens to always bid between 0 and 1 , and that players 1 and 2 have values that are always at least 2 . Then, each of player 1 and player 2 would like any bid he makes to always exceed any bid by the other player. So, optimal bids (at least in some scenarios) are unbounded.

For an example in which optimal buy bids may not be bounded from below, consider an auction in which players each demand two units, and in which $k$ units are for sale. The price is a convex combination of the $k$ and $(k+1)$ th highest bids, with the weight on the $k$ th being a strictly increasing function of the average bid. Then, a bidder with a low second value might well find it optimal to submit a first bid near his first value, but make his second bid arbitrarily negative.

${ }^{16}$ While treated identically to other bid vectors, $b_{0}$ can be thought of as 0 's reserve price vector. In order to have player 0 not participate in the auction at all (for instance in a double auction) we can simply set $e_{0}=0$ and $b_{0 h}=\underline{b}$ for all $h$, in which case all of 0 's bids are noncompetitive.

${ }^{17} \mathrm{Secret}$ reserve prices are handled by having player 1 have the only positive endowment, so that player 1 is the seller and his bid is the secret reserve.

${ }^{18}$ Note that we are considering the game having fixed $b_{0}$, and not the game in which $b_{0}$ is chosen strategically. It follows from Theorem 2 of JSSZ that the set of equilibria of the game defined by $b_{0}$ with omniscient tie-breaking is upper hemi-continuous in $b_{0}$. By Theorem 9 , every such equilibrium is an equilibrium under standard tie-breaking. Hence, the set of Nash equilibria of the game induced by $b_{0}$ is upper hemi-continuous. It follows that there is also an equilibrium of the game in which buyer 0 chooses $b_{0}$ according to some objective.
} 
continuously as a function of the vector of submitted bids. Thus, there are continuous functions $t_{i}:\{0, \ldots, \ell\}^{n+2} \times \mathcal{B} \rightarrow \mathbb{R}$, such that $i$ 's payment is $t_{i}(h, e, b)$ in the case where the bid profile is $b$, the endowment vector is $e$, and he receives $h$ objects. $^{19}$

Note that the assumption of continuity here is consistent with the discontinuities present in an auction setting. This is because $t_{i}(h, e, b)$ only says what $i$ would pay if $i$ were to receive $h$ objects. A change in bids can still change how many objects $i$ gets, say from $h$ to $h+1$, and thus can still lead to a discontinuous change in payments. For example, in a first price auction, $t_{i}(1,0, b)=b_{i}$ and $t_{i}(0,0, b)=0$, both of which are clearly continuous even though the payment as a function of bids accounting for ties and changes in the number of objects received is not continuous.

Typically (but not always, as illustrated by Example 4$), t_{i}(h, e, b)$ will have the same sign as $i$ 's net trade, $h-e_{i}{ }^{20}$

\subsubsection{Payoffs}

Players evaluate the outcome of the auction via von Neumann-Morgenstern utility functions. This allows for risk-averse, risk-loving, or any of a variety of other sorts of preferences.

ASSUMPTION 6 (Expected Utility): Player $i \in N$ has a von NeumannMorgenstern utility function $U_{i}$ over her net payoff. $U_{i}$ is continuous, strictly increasing and has a first derivative that is bounded away from 0 and $\infty$.

Assumption 6 implies that there exists $\Lambda<\infty$ such that $U^{\prime}(x) / U^{\prime}(y)<\Lambda$ for all $x$ and $y$.

So, a player's utility when receiving $h$ objects in the final allocation when the bid vector is $b$ and the endowment is $e$ is described by

$$
U_{i}\left(\left(\sum_{h^{\prime} \leq h} v_{i h^{\prime}}\right)-t_{i}(h, e, b)\right)
$$

where $U_{i}$ is a continuous von Neumann-Morgenstern utility function.

\footnotetext{
${ }^{19}$ The way in which we have defined $t$ includes a specification of payments for $b$ and $h$ where in fact it is not consistent for $i$ to receive $h$ objects given $b$. This is a notational convenience.

${ }^{20} \mathrm{An}$ important point about the way in which we have formulated the payment rule is that player $i$ 's payment can depend on his own allocation but does not further depend on other players' allocations, such as which players other than $i$ won. Without this assumption, an entirely new and tricky set of discontinuities arises. For example, even though player $i$ might happen not to be involved in a tie, small changes in his bid might affect which of two opponents wins a tie (remember that an omniscient tie-breaking rule allows for this possibility). If this results in a change in the payment rule $i$ faces, then his payoffs may change discontinuously despite the fact that his bid is not involved in any ties.
} 


\subsection{Examples and a Preview of Our Results on a Narrower Class of Auctions}

The general development that follows is involved given the breadth of the class of auctions handled and the attention paid to allocations and tie-breaking rules. Thus, we offer some examples of auctions covered by our existence results and a preview of some of the results for a narrower class of auctions. This class still includes most standard auction formats.

Although our statements here should be clear, we refer the reader to the subsequent sections for the formal and more general statements of our results.

We emphasize that in all of the examples that follow there is no assumption about symmetry of the distribution of the players' endowments, valuations, or utility functions.

(i) A Standard First Price Single Unit Auction. In terms of our definitions and notation this is expressed as follows. There is one object sold by player 0 , and so $\ell=1$ and the distribution over endowments is such that $\operatorname{Pr}(\{e=(1,0, \ldots, 0)\})=1$. The payments are such that an agent pays his bid if he wins an object $\left(t_{i}(1, e, b)=b_{i 1}\right)$ and nothing otherwise $\left(t_{i}\left(0, e_{i}, b\right)=0\right)$. The reserve price is $b_{01}=0$. Let $C \equiv\left\{i \in N \mid b_{i 1} \geq b_{j 1}\right.$ for all $\left.j \in N \cup\{0\}\right\}$ (the set of players who submitted the highest bid). Then, the allocation rule gives the object to player $i \in N$ with probability $1 / \# C$ if $i$ is in $C$ and 0 otherwise. If $C$ is empty (so that no player other than 0 bids at least 0 ), then player 0 retains the object.

Note that because the seller submits a bid at 0 , a negative bid by any other player never wins. Hence, such bids are simply a way for players to express that they have no interest in winning.

(ii) A Standard First Price Single Unit Auction with a Known Reserve Price, $r \geq 0$. This is the same as Example 1, except that player 0 sets a reserve price $b_{01}=r$.

(iii) A Single Unit Vickrey (Second Price) Auction. This is as in (1) or (2), except that the payment rule for a winning bidder changes to $t_{i}(1, e, b)=b^{2}$, where $b^{2}$ is the second highest bid submitted (including the reserve price $\left.b_{01}=r\right)$.

(iv) An Unfair Auction. This is the same as any of the above examples except that some players pay only some fraction of the payments indicated above when they win while other players' payments are unchanged.

(v) An Auction with Entry Costs (and a Reserve Price). Let $c \geq 0$ be the entry cost incurred by a player wishing to make a bid, where the decision of whether and how to bid is made without knowing other players' entry decisions. This is handled in our model as follows. The setting is as in (1), (2), or (3), except that $t_{i}(0, e, b)=c \min \left\{b_{i 1}+1,1\right\}$ and $t_{i}(1, e, b)=c \min \left\{b_{i 1}+1,1\right\}+b_{i 1}$ for the first price version (with $\left.t_{i}(1, e, b)=c \min \left\{b_{i 1}+1,1\right\}+b^{2}\right\}$ for a second price auction).

The form of $t_{i}(0, \cdot)$ is chosen so that the function is continuous and satisfies our assumptions. Effectively, sending a bid of -1 means that the player stays out of the auction and does not pay the cost $c$, whereas sending any bid $b_{i} \geq 0$ 
incurs the cost $c$ of participating. The remaining bids between -1 and 0 are bids that would never be used in equilibrium since they cannot win an object (given a reserve price of $r \geq 0$ ) and yet would incur some bidding cost. Thus, the presence of the bids that lie above -1 and below 0 is just a technical device in this example.

(vi) An All Pay Auction (and Various Implementations of the War of Attrition). A first price all pay auction is the same as in (1) except that $t_{i}(0, e, b)=$ $t_{i}(1, e, b)=b_{i}$. In the standard war of attrition the winner pays the second highest bid and so $t_{i}(1, e, b)=b^{2}$ as in (2), while $t_{i}(0, e, b)=b_{i}$.

(vii) A First Price Procurement Auction. Here $\ell=1$ and player 0 has $e_{0}=0$. Thus, setting $b_{01}>0$ represents the maximum amount that 0 will pay for an object. Each player $i>0$ has $e_{i}=1$. The lowest bidder among $i>0$ sells an object to player 0 provided the bid is no more than $b_{01}$ (with ties among players $i>0$ broken in any way). The payment if an object is sold by $i$ to 0 is $t_{i}(0, e, b)=$ $-b_{i 1}$. That is, the buyer pays $b_{i 1}$ to the winning seller. Otherwise payments are 0 . The obvious variation leads to a second price version of a procurement auction. ${ }^{21}$

(viii) A Multi-Unit Discriminatory (Pay Your Bid) Auction. Take $\ell>1$ and $\operatorname{Pr}(\{e=(\ell, 0, \ldots, 0)\})=1$. The top $\ell$ bids are declared winners, and payments are $t_{i}(h, e, b)=\sum_{w=1}^{h} b_{i w}$.

(ix) A Multi-Unit Uniform Price Auction. As in (8), except that winning players pay the $(\ell+1)$ th highest bid for each unit they acquire, so $t_{i}(h, e, b)=$ $h b^{\ell+1}$, where $b^{\ell+1}$ is the $(\ell+1)$ th highest bid. 22,23

(x) A Standard Double Auction. Players 1 through $n_{b}$ are potential buyers having $e_{i}=0$. Players $n_{b}+1$ through $n=n_{b}+n_{s}$ are potential sellers having $e_{i}=1$. Ties between a buyer and a seller are broken in favor of trade, and ties among buyers or among sellers are broken randomly. Let $p=\left(b^{\prime}+b^{\prime \prime}\right) / 2$, where $b^{\prime}$ is the $n_{s}$ th highest bid, and $b^{\prime \prime}$ the $\left(n_{s}+1\right)$ th. Then, $t_{i}(0, e, b)=-p e_{i}$, while $t_{i}(1, e, b)=p\left(1-e_{i}\right)$.

(xi) A Generalized Double Auction. Players 0 through $n$ draw a realization of $\left(e_{i}, v_{i}\right)$, and submit bid vectors. Objects are allocated to the $\sum_{i=0}^{n} e_{i}$ highest players, with tie-breaking as in (9). Let $t_{i}(h, e, b)=p\left(h-e_{i}\right)$, where $p$ is a weakly increasing and continuous function of the $\left(\sum_{i=0}^{n} e_{i}\right)$ th and $\left(\sum_{i=0}^{n} e_{i}+1\right)$ th highest bids. Note that players may turn out to be buyers or sellers, even for a given realization of their own type vector, depending on how their bid vector compares to those of other players.

\footnotetext{
${ }^{21}$ Extensions to multi-unit procurement auctions are also easily handled.

${ }^{22}$ There are many variations on ways to select the price paid, including some that ensure that a player's losing bid does not end up setting the price he pays for his winning bids (such as that suggested by Vickrey (1961)). The particulars of how the price is chosen and even whether it differs across players will not matter, as our theorem will apply in any case.

${ }^{23}$ Examples (8) and (9) cover the class of private value auctions examined in Swinkels (1999, 2001) and Jackson and Kremer (2004).
} 
(xii) A Double Discriminatory Auction. This is the same as (11), except for the payments. If a player ends up as a net buyer with $h$ objects, he pays $\sum_{h^{\prime}=e_{i}+1}^{h} b_{i h^{\prime}}$. If $i$ ends up as a net seller, he receives $\sum_{h^{\prime}=h+1}^{e_{i}} b_{i h^{\prime}}$. The auctioneer (player 0) pockets the difference.

THEOREM 2: Each of the auctions described above has an equilibrium in distributional strategies that have support in the closure of the set of undominated strategies.

In one-sided auctions (or more generally, any auction where there is a nonstrategic seller with a reserve below $\bar{v}$ ), the equilibrium above will automatically have trade. When there is no nonstrategic seller, this is less clear. For example in a double auction, there may exist degenerate equilibria where all sellers bid at the top of the support of values and buyers bid at the bottom.

Existence of equilibria with a positive probability of trade is guaranteed with two additional assumptions. First, we require that changing one player's type does not alter the support of types for another. In particular, we assume that the Radon-Nikodym derivative of $P$ with respect to $\prod_{i} P_{i}$ is always positive. Second, we assume that there is some competition for gains from trade. It is enough to have the support of buyer and sellers' valuations overlap for all $h$ and to have either at least two buyers or at least two sellers. A weaker condition is described in Section 5.

THEOREM 3: Under the above-mentioned assumptions, each of the auctions described above has an equilibrium that has support in the closure of the set of undominated strategies and has a positive probability of trade.

\subsection{The General Class of Auctions: Allocations and Tie-Breaking Rules}

We now return to the formal definitions of allocations and tie-breaking, which are needed in the full statement of our results and to complete a description of the class of auctions that we consider.

\section{Allocations}

An allocation is a vector $a \in\{0, \ell\}^{n+1} \equiv A$. The component $a_{i}$ is the number of objects that are allocated to player $i$.

\section{Consistent allocations}

An allocation is consistent with vectors of endowments and bids $(e, b)$ if

$$
\sum_{i=0}^{n} a_{i}=\sum_{i=0}^{n} e_{i}
$$


and

$$
\left\{b_{j h^{\prime}}>b_{i h} \text { and } a_{i} \geq h\right\} \Rightarrow a_{j} \geq h^{\prime} .
$$

The first condition is simply a balance condition, requiring that all objects be accounted for. Note that this allows for the possible interpretation that objects that are allocated to the 0 player might be "unsold," for instance in the case where player 0 is the only seller in an auction.

The second condition simply says that if $i$ receives at least $h \geq 1$ objects and $j$ 's $h^{\prime}$ th bid exceeds $i$ 's $h$ th, then $j$ must get at least $h^{\prime}$ objects. Thus, higher bids are given priority over lower bids in allocating objects. ${ }^{24}$

Let $C(e, b) \subset A$ denote the set of consistent allocations given endowment and bid vectors $(e, b)$.

Ties

Say that there is a tie given $(e, b)$ if there exist $a$ and $a^{\prime}$ in $C(e, b)$ such that $a \neq a^{\prime}$. Say the tie is at $b^{*}$ if

$$
\begin{aligned}
& \#\left\{i, h \mid b_{i h}>b^{*}\right\}<\sum_{i=0}^{n} e_{i} \text { and } \\
& \#\left\{i, h \mid b_{i h} \geq b^{*}\right\}>\sum_{i=0}^{n} e_{i} .
\end{aligned}
$$

So, in the event of a tie at $b^{*}$, all bids above $b^{*}$ are filled, but there is some discretion in to whom to allocate objects at $b^{*}$. Thus, for instance, it is not a tie if there are two objects for sale, bidder one has the highest bid, and bidder two has two identical bids that are the next-highest. Here bidder two has the "tied" bids, but will always get one object in any allocation.

\section{Tie-breaking rules}

As discussed in the Introduction, we prove existence for a very wide class of tie-breaking rules, including some fairly strange ones. In particular, we allow for the possibility that the auctioneer uses more information than just bids and endowments in determining allocations.

An omniscient tie-breaking rule is a (measurable) function $o: \Theta \times \mathcal{B} \rightarrow \Delta(A)$ such that $o(e, v, b)$ places probability one on the set of consistent allocations

\footnotetext{
${ }^{24}$ In some auctions, some players enjoy a special status. For example, some of the PCS auctions subsidized bids by minority owned firms (see Cramton (1995)). One way of implementing this would be to declare the minority firm a winner if its bid is at least, say, $2 / 3$ of the highest bid. We instead include asymmetries by insisting on the highest bid winning, but allowing payment rules to differ, so that, for example, the minority firm pays only $2 / 3$ of its submitted bid.
} 
$C(e, b)$. We let $o(e, v, b)[a]$ denote the probability of allocation $a$ under $o$ at $(e, v, b)$, and $o_{i}(e, v, b)[h]$ denote the probability that $i$ is allocated $h$ objects under $o$ at $(e, v, b){ }^{25}$

Given the requirement of consistency, $o$ only has any discretion where there are tied bids, and hence the term "tie-breaking rule" is appropriate.

Let standard tie-breaking be the particular tie-breaking rule that is defined as follows. Consider a tie at $b^{*}$. First, allocate an object to each bid that is strictly above $b^{*}$. Next, allocate an object with equal probability to each player who has an unfilled buy bid at $b^{*}$. Repeat until all objects are gone, or until there are no unfilled buy bids at $b^{*}$. At this point, iteratively allocate any remaining objects one at a time with equal probability to those players who have an unfilled sell bid at $b^{*}$.

The two key aspects of standard tie-breaking are first that the rule is trademaximizing, and second that a bidder's chance of winning an $h$ th object at $b^{*}$ does not depend on $i$ 's other bids. This would be false, if, for example, one simply randomly assigned remaining objects equiprobably over all bids at $b^{*}$, as then an $(h+1)$ th bid of $b^{*}$ would increase the chance that $i$ receives object $h$.

While we were led to consider omniscient tie-breaking rules for their use as an intermediate step in the proof of existence, it also strikes us that there may be situations in which tie-breaking that depends on more than just bids might be appropriate. For example, the government may have objectives beyond those of revenues that would push them to favor one player over another in the event of a tie. In this value setting, we will see that ties never occur anyway, so any extra information available to the auctioneer turns out to be irrelevant. It is an open question whether meaningful ties occur in other settings, and whether the possibility of favoritism, etc., would have an interesting effect in those settings.

\section{Competitive ties and trade maximization}

It should be noted that not all ties are the same. On the one hand, is a situation in which two buyers are tied at a given bid, and only one of them receives an object. As we will show, at least one player will always have an incentive to deviate in this situation. Consider on the other hand, a situation in which a single buyer and a single seller make a tied bid, but the object is transferred from buyer to seller at a price under which both are happy to trade. Here, $C(e, b)$ has more than one element, since it is also consistent for trade not to occur. But, since the object is actually transferred, there is no incentive for either player to change their bid.

\footnotetext{
${ }^{25}$ Imagine the auctioneer had access to some other information, possibly correlated with player type, but unobservable to the players at the time they bid. Allowing the auctioneer to also condition on this information in breaking ties would not expand the set of equilibria beyond those achieved with omniscient tie-breaking: from the point of view of the players, this is equivalent to the auctioneer simply randomizing in a way that depends on the types and bids of the players.
} 
Let us say that $(e, b)$ has a competitive tie if there exists a $p$ such that the number of buy bids that are greater than or equal to $p$ is not the same as the number of sell bids that are less than or equal to $p$.

It turns out that while equilibrium conditions will naturally rule out competitive ties, noncompetitive ties may occur in equilibrium. In particular, it is possible that a buyer and seller have a tied bid. As long as trade always occurs in this situation, this is not inconsistent with equilibrium. This is captured in the following condition.

A tie-breaking rule $o$ is trade-maximizing at $(v, e, b)$ if the rule does not specify an allocation in which one player has an unfilled buy bid at $b$ and another has an unaccepted sell bid at $b .{ }^{26} \mathrm{~A}$ tie-breaking rule $o$ is trade-maximizing if it is trade-maximizing at every $(v, e, b)$.

We will be working with distributional strategies (see Section 3.5 for details). Given a probability measure $m$ on $\Theta \times \mathcal{B}$, say that the rule $o$ is effectively trade-maximizing if it is trade-maximizing on a set of $(v, e, b)$ having measure 1 under $m$. So, given the way in which types are drawn and players randomize over bids, the probability that there is a non-trade-maximizing tie is zero.

The next example illustrates the importance of the trade-maximization in our invariance result.

EXAMPLE 4: There is a buyer with a valuation for a single unit uniformly drawn from $[3,4]$, and a seller with a valuation for a single unit for sale and value uniformly drawn from $[0,1]$, where values are independent across players. The price is the midpoint between the bids.

If in the event of a tie between a buy and sell bid the auction mechanism specifies that trade should occur, then it is an equilibrium for both players to bid 2, and for trade to always occur if both players bid 2 . This rule is effectively trade-maximizing. If instead the auction mechanism specifies that in the event of a tie, trade occurs with a probability $\rho<1$, then this is no longer an equilibrium. Now a buyer would benefit by slightly raising his bid, or a seller would benefit from slightly lowering her bid. In fact, now there is no longer any equilibrium in which trade always occurs. To see this, suppose the contrary. Then, almost every bid by the buyer must exceed almost every bid by the seller. But then, a bid near the bottom of the support of buyer's bids wins almost always, and so does strictly better than a higher bid. Thus, the buyer must be making the same bid $b_{B}$, regardless of valuation. Similarly, the seller must be making the same bid $b_{S}$, regardless of value. Suppose that $b_{S}<b_{B}$. Then, a seller can raise his bid and still almost always sell at a better price, a contradiction. Hence $b_{S}=b_{B}=p$ for some $p$. But this is a contradiction since the supposition is that trade occurs with probability $\rho<1$ at a tie.

\footnotetext{
beat it.

${ }^{26}$ This includes player 0 . So, for example, it is only necessary to meet a reserve price, not strictly
} 


\section{Payments and bids}

We need to say something about how bids determine payments. First, we require that for any given allocation, a player who is a net buyer is weakly better off to have submitted lower bids, and a player who is a net seller is weakly better off to have submitted higher bids. Of course, this is holding the allocation constant. Such a change in bid may well result in the loss of a profitable trade. Second, we will require that if one is a net seller, one's buy bids do not matter, and vice versa.

AsSUMPTION 7 (Monotonicity): For any $i$, and $h \geq e_{i}, t_{i}(h, e, b)$ is nondecreasing in $b_{i h^{\prime}}$ for $h^{\prime}>e_{i}$ and constant in $b_{i h}$ for $h^{\prime} \leq e_{i}$; and if $h<e_{i}$, then $t_{i}(h, e, b)$ is nonincreasing in $b_{i h^{\prime}}$ for $h^{\prime} \leq e_{i}$, and constant in $b_{i h^{\prime}}$ for $h^{\prime}>e_{i}$.

Note that the condition does not impose any requirements about how a player's payment depends on the bids of others.

Having buy payments be independent of sell bids, and vice versa, is useful in our weak domination arguments (for instance Lemma 5), and also in establishing the existence of positive trade (Theorem 15).

\section{Payments at ties}

The following condition on payments is critical to our results. It says that one's marginal payment when one's $h$ th bid is involved in a tie is a function only of $e_{i}, h$, and $b_{i h}$. One's other bids, and the details of with how many other players one is tied, and what their other bids were, are irrelevant.

AsSUMPTION 8 (Known Marginal Transfers at Ties): For all $i, h$, and $e_{i}$, there is $p_{\text {ihe }}:[\underline{b}, \bar{b}] \rightarrow \mathbb{R}$ such that if $(h, e, b)$ is such that there is a tie at $b_{i h}$, then $t_{i}(h, e, b)-t_{i}(h-1, e, b)=p_{i h h_{i}}\left(b_{i h}\right)$.

This condition is generally satisfied and easy to check. For instance, for discriminatory auctions, uniform price auctions, and all double auctions (where the price is set in the range of market clearing prices for the submitted demand and supply curves), $p_{i h}\left(b_{i h}\right)=b_{i h} .^{27,28} \mathrm{It}$ is also satisfied for an all pay auction, where the difference in payments does not depend on whether the player gets an object and so $p_{i h}\left(b_{i h}\right)=0$. It is not satisfied for a third price auction for a single unit, since then, even if the first two bids are tied, the price paid may vary depending on the third bid.

\footnotetext{
${ }^{27}$ Note that in a double auction where a player has a single unit to sell and $b_{i}$ is in a tie, $t_{i}\left(0,1, b_{i}\right)=-b_{i}$ and $t_{i}\left(1,1, b_{i}\right)=0$. So, $p_{i 1}=0-\left(-b_{i}\right)=b_{i}$.

${ }^{28}$ This also holds for Vickrey auctions where the price is the highest bid among other players. Note that in the case of a tie, since that requires that there must be some discretion in the awarding of objects, the highest nonwinning bid among others must also be tied.
} 
Our invariance results do not hold when marginal transfers might be decreasing in $h$. This effectively induces a volume discount, which creates much the same phenomenon as an upward sloping demand curve: at some bid vectors where the player's two bids are tied, the player will be unhappy to win a single object, but happy to win two.

ASSUMPTION 9 (Monotonic Marginal Payments): For each i, $e_{i}, p_{i h e_{i}}(b)$ is nondecreasing in $h$.

This assumption is trivially satisfied where $p_{\text {ihe }}(b)=b$ or $p_{i h e_{i}}(b)=0$.

To see an example where in the absence of such a condition one might get a rather odd equilibrium and how this might depend on the tie-breaking rule, consider a case in which each of three players has marginal value 4 for two objects. Half the time, one object is available, and half the time, four. Assume that payment rules are such that, when there is a tie at a bid of $b^{*}$, a player pays 6 for a first unit and 0 for a second. Then, it is an equilibrium for all three players to bid $\left(b^{*}, b^{*}\right)$ always, as long as tie-breaking is that when there is a tie and a single object, each player receives the object one third of the time, while when there is a tie and four objects, each player receives two objects with probability two thirds, and no object with probability one third. Then, by submitting $\left(b^{*}, b^{*}\right)$, a player earns

$$
\frac{1}{2}\left[\frac{1}{3}(4-6)+\frac{2}{3}(8-6)\right]=\frac{1}{3} .
$$

Consider any deviation in which a player raises either his first bid or both bids. By the rules of consistent allocations, this must have him always being allocated the object when there is only one, and paying at least 6 . In the most favorable case, it always has him also win two objects whenever there are four available. Hence, he earns at most

$$
\frac{1}{2}(4-6)+\frac{1}{2}(8-6)=0
$$

from this deviation. Lowering just the second bid results in sometimes winning a first object at a loss, and never winning a second object. Lowering both bids results in payoff 0 . The problem here is that the player would effectively like to raise his second bid and lower his first, which is infeasible.

\subsection{Strategies and Equilibrium}

Given the definitions from the previous subsections, an auction is a specification of $\left(P, o, t, b_{0}\right)$. That is, an auction consists of a probability measure, a tie-breaking rule, a payment rule, and a reserve price vector. In what follows, in some cases it will be clear that these are given and we omit mention of them. 
We now turn to formal definitions of the game induced by the auction in terms of strategies and equilibrium.

We write $i$ 's expected utility given a (possibly omniscient) tie-breaking rule $o$, payment rule $t$, bid profile $b$, valuation vector $v$, and endowment profile $e$ as

$$
u_{i}(o, t, b, e, v)=\sum_{a_{i}=0}^{\ell} o_{i}(e, v, b)\left[a_{i}\right] U_{i}\left(\left(\sum_{h \leq a_{i}} v_{i h}\right)-t_{i}\left(a_{i}, e, b\right)\right) .
$$

\section{Strategies}

A (distributional) strategy for player $i$ is a (Borel) probability measure $m_{i}$ on $\mathcal{B}_{i} \times \Theta_{i}$ that has a marginal distribution of $P_{i}$ on $\Theta_{i}$.

See Milgrom and Weber (1985) for discussion of distributional strategies.

Given a profile of distributional strategies $m_{1}, \ldots, m_{n}$, player $i$ 's expected payoff can be written as:

$$
\begin{aligned}
& \pi_{i}\left(m, P, o, t, b_{0}\right) \\
& \quad=\int u_{i}(o, t, b, e, v) d m_{1}\left(b_{1} \mid e_{1}, v_{1}\right) \cdots d m_{n}\left(b_{n} \mid e_{n}, v_{n}\right) d P(e, v) .
\end{aligned}
$$

When some of the arguments are fixed, we omit them from the notation, and for instance write $\pi(m, o)$ when all but the tie-breaking rule is fixed.

\section{Equilibrium}

A profile of distributional strategies $m_{1}, \ldots, m_{n}$ is an equilibrium for auction $\left(P, o, t, b_{0}\right)$ if

$$
\pi_{i}\left(m, P, o, t, b_{0}\right) \geq \pi_{i}\left(m_{-i}, \widehat{m}_{i}, P, o, t, b_{0}\right)
$$

for all $i$ and strategies $\widehat{m}_{i}$.

\section{Weak dominance}

As we wish to prove existence of equilibria that satisfy a refinement that will rule out some trivial equilibria, we establish that players use strategies in the closure of the set of undominated strategies. The formal definitions are as follows.

Say that bid vector $b_{i}$ is weakly dominated at $e_{i}, v_{i}$ by $b_{i}^{\prime}$ if

$$
u_{i}\left(o, t, b_{-i}, b_{i}^{\prime}, e, v\right) \geq u_{i}\left(o, t, b_{-i}, b_{i}, e, v\right),
$$

for any $e_{-i}, v_{-i}, b_{-i}$, with strict inequality for at least one such profile, where $o$ is standard tie-breaking. We say that $b_{i}$ is undominated at $e_{i}, v_{i}$ if it is not weakly dominated by any other bid. 
Note that we include the bid of player 0 in this definition, which is not completely standard, as, at the time that a player submits his bid, he already knows $b_{0}$. This provides for a stronger result and actually simplifies the proofs.

It is worth discussing why our definition of weak dominance is relative to standard tie-breaking. With nonstandard tie-breaking, some pretty odd behaviors are undominated, especially in the multiple unit demand case. Consider an example where two players each value two units. The auction rule is that all objects are sold at the lowest winning bid. Most of the time, 2 objects are available. Occasionally, there is only 1 . Finally, player 2 always submits two bids of 3 . The tie-breaking rule, for whatever reason, is that if there is a tie at 3, and player 1's first bid is 6 , then both objects go to player 1. If player 1's first bid is anything else, the second object is allocated at random. Then, when player 2 has value vector $(5,4)$, it is undominated for him to bid $(6,3)$, even though his first bid is higher than his first value. Under standard tie-breaking, of course, one's first bid is irrelevant to the probability that one's second bid is filled if one is involved in a tie, and such a bid vector is indeed weakly dominated. Although this example is clearly artificial, either explicitly or implicitly, either approach to existence, via either JSSZ or Reny, requires us to admit this type of thing as a possibility. Thus to really have the appropriate bite on weakly dominated strategies, we rule them out under standard tie-breaking rules.

It is well known that existence of equilibrium in games with continuous action spaces may require the use of weakly dominated actions. An example is equilibrium in a Bertrand game with symmetric constant marginal costs. As such a game has analogs in the auction setting, we cannot meaningfully require that a distributional strategy put weight 0 on weakly dominated bids. It is, however, coherent to require that the distributional strategy put probability 1 on the closure of the set of nonweakly dominated bids.

To formalize this, let $W_{i}^{\prime} \subset \Theta_{i} \times \mathcal{B}_{i}$ be the set of $e_{i}, v_{i}, b_{i}$ such that $b_{i}$ is undominated for $i$ given $e_{i}, v_{i}$. Let $W_{i}$ be the closure of $W_{i}^{\prime}$.

ASSUMPTION 10 (Undominated Strategies): For each player $i \in N$, there is a measurable map $\omega_{i}: \Theta_{i} \times \mathcal{B}_{i} \rightarrow \Theta_{i} \times \mathcal{B}_{i}$ such that for each $\left(e_{i}, v_{i}, b_{i}\right)$, $\omega_{i}\left(e_{i}, v_{i}, b_{i}\right)=\left(e_{i}, v_{i}, b_{i}^{\prime}\right)$ in $W_{i}$ where $b_{i}^{\prime}=b_{i}$ if $\left(e_{i}, v_{i}, b_{i}\right) \in W_{i}$, and $b_{i}^{\prime}$ weakly dominates $b_{i}$ given $\left(e_{i}, v_{i}\right)$ if $\left(e_{i}, v_{i}, b_{i}\right) \notin W_{i}$.

Assumption 10 states that one can, in a measurable way, identify bids so that whenever $\left(e_{i}, v_{i}, b_{i}\right) \notin W_{i}$, then $b_{i}$ is replaced by a bid that weakly dominates it and results in an element of $W_{i}$.

Of course, for this to be satisfied, one needs to know that $W_{i}$ is in fact nonempty relative to each $\left(e_{i}, v_{i}\right)$. For general games with continuum action spaces, this need not be so. Consider a game with action space [0,1], and payoffs equal to action for all actions less than 1, but equal to -1 for action 1 . Then, all actions are weakly (in fact strictly) dominated. The question in the 
auction setting is whether similar things might arise, especially once one considers what happens to payoffs at ties (where it is easy to construct bid vectors relative to which there is no best response).

We have not found a sensible auction-like example where Assumption 10 fails. For example, in a first price auction (or a discriminatory multiple unit auction), any bid less than value is undominated: any higher bid may simply result in bidding more in situations where one might already have won, while any lower bid may result in the loss of a profitable purchase. $W$ thus includes all bids in which one bids at or below value. Similarly, in a second price auction, a bid equal to value is not weakly dominated. And, in either case, replacing buy bids above value (or sell bids below value) by bids at value is clearly measurable. ${ }^{29}$ In an all pay auction, a bid of zero is not weakly dominated, and replacing bids above value by 0 is again clearly measurable. Using these two ideas, it is easy to check that Assumption 10 is satisfied for all the auctions discussed in Section 3.3.

A useful observation is the following:

LEMMA 5: Under Assumptions 1-10, given $\left(e_{i}, v_{i}\right)$, let $b_{i}$ be any bid vector such that $p_{\text {ihe }}\left(b_{i h}\right)<v_{\text {ih }}$ for some $h \leq e_{i}$, or $p_{\text {ihe }}\left(b_{i h}\right)>v_{\text {ih }}$ for some $h>e_{i}$. Then $\left(e_{i}, v_{i}, b_{i}\right) \notin W_{i}$.

That such a bid vector is weakly dominated at $\left(e_{i}, v_{i}\right)$, so that $\left(e_{i}, v_{i}, b_{i}\right) \notin W_{i}^{\prime}$ can be seen as follows. If one raises a sell bid where $p_{i h e_{i}}\left(b_{i h}\right)<v_{i h}$, then the only change can be to either sell one less object, which originally sold at a loss, or to raise the market price as a seller, either of which benefits the player. By Assumption 7, there can be no change if one was a net buyer before the change in bid. A similar argument applies when one lowers a buy bid where $p_{i h e_{i}}\left(b_{i h}\right)>v_{i h}$. Note that the same argument will be true for nearby $\left(e_{i}^{\prime}, v_{i}^{\prime}, b_{i}^{\prime}\right)$. Hence, a neighborhood of $\left(e_{i}, v_{i}, b_{i}\right)$ is outside of $W_{i}^{\prime}$, and so $\left(e_{i}, v_{i}, b_{i}\right) \notin W_{i}$. A slightly more detailed proof appears in the Appendix.

We say that a profile of distributional strategies $m$ is undominated* if each $m_{i}$ places probability one on $W_{i}$.

\section{INVARIANCE AND EXISTENCE}

We now state our first main result.

THEOREM 6: If an auction $\left(P, o, t, b_{0}\right)$ satisfies Assumptions 1-10 and $o$ is trade-maximizing, then it has at least one undominated* equilibrium. Moreover, if $m$ is such an equilibrium, then the probability of competitive ties under $m$ is 0 ; and $m$ remains an equilibrium under any omniscient and effectively trade-maximizing tie-breaking rule, including standard tie-breaking.

\footnotetext{
${ }^{29}$ In an unfair first price auction in which player 1 pays, say, $2 / 3$ of his bid, one would replace bids above $3 v_{i} / 2$ by bids of $3 v_{i} / 2$.
} 
Our route for the proof of Theorem 6 is as follows. We begin with invariance: we show that any undominated* equilibrium must have zero probability of any player being involved in a competitive tie and would also be an equilibrium if we changed the method of tie-breaking to any other effectively trade-maximizing omniscient tie-breaking rule. Using invariance, if we can establish existence of an equilibrium in nonweakly dominated strategies for some omniscient tie-breaking rule, this implies existence of (the same) equilibrium under any tie-breaking rule, omniscient or standard. This second step is fairly easily established via either of two results, either JSSZ or Reny.

The discussion of invariance appears in Section 4.1. The step from invariance to existence is in Section 4.2, with additional details in the Appendix. Those not interested in the proof can proceed directly to Section 5 to find results establishing the existence of equilibria with a positive probability of trade, an important issue in auctions such as double auctions.

\subsection{Invariance}

The fact that ties are the critical worry for establishing existence of equilibrium follows from the fact that ties are the only potential points of discontinuity. So, intuitively, if we establish that players prefer to avoid ties, then we show that the discontinuities are not important, which in turn allows us to establish the existence of equilibrium. Let us go right to the heart of the matter.

LEMMA 7: Consider any auction $\left(P, o, t, b_{0}\right)$ satisfying Assumptions $1-10$ and any profile of strategies $m$. For each $\varepsilon>0$ and any bidder $i \in N$ there exists $m_{i}^{\prime}$ within $\varepsilon$ of $m_{i}{ }^{30}$ such that $m_{-i}, m_{i}^{\prime}$ is tie-free for $i^{31}$ and $\pi_{i}\left(m_{-i}, m_{i}^{\prime}\right) \geq$ $\pi_{i}\left(m_{-i}, m_{i}\right)-\varepsilon$.

Lemma 7 shows that for any profile of strategies, any player can find a closeby strategy that does not involve any ties and does nearly as well as her original strategy. ${ }^{32}$

Note that Lemma 7 does not put any requirements on $o$ or on $m$, and so it allows for tie-breaking that is not trade-maximizing and for strategies that are in weakly dominated strategies.

LEMMA 8: Fix an auction $\left(P, o, t, b_{0}\right)$ satisfying Assumptions $1-10$. Let $m$ be undominated ${ }^{*}$, and either have a positive probability of ties where o is not trade-

\footnotetext{
${ }^{30}$ Use the topology of weak convergence.

${ }^{31}$ That is, $m_{-i}, m_{i}^{\prime}$ leads to a probability 0 of $i$ being involved in a tie.

${ }^{32}$ It is important to remark that this is not the same as establishing better-reply-security as defined by Reny (1999). Better-reply-security does not hold here, as we discuss in more detail below. We are not considering all payoffs that may be reached in the closure of the graph of the game, only ones that directly correspond to the strategies in question.
} 
maximizing or a positive probability of competitive ties. Then, there exists some bidder $i \in N$ and a strategy $m_{i}^{\prime}$ such that $m_{-i}, m_{i}^{\prime}$ is tie-free for $i$ and

$$
\pi_{i}\left(m_{-i}, m_{i}^{\prime}, P, o, t, b_{0}\right)>\pi_{i}\left(m, P, o, t, b_{0}\right) .
$$

Lemma 8 shows that for any profile of strategies placing probability one on the closure of the set of undominated strategies, but involving a positive probability of competitive ties or noncompetitive ones that are not trade-maximizing, some player has an improving deviation. This implies that if there exists an undominated* equilibrium, then it must not involve any such ties.

The proofs of Lemmas 7 and 8 appear in the Appendix. The idea behind Lemma 7 is fairly straightforward. Essentially, one bumps bids $b_{i h}$ for which $v_{i h}>p_{i h e_{i}}\left(b_{i h}\right)$ up slightly and bids for which $v_{i h} \leq p_{i h e_{i}}(b)$ down slightly in such a way as to avoid bids made by other players with positive probability. Any change in trade this brings about is at most slightly unprofitable. For example, if a buy bid is bumped up, and wins an extra object, then the payment for the object is approximately $p_{i h e_{i}}\left(b_{i h}\right)$. Since the change in bids is small, the profit of the player when his allocation does not change is also little affected. The detailed proof is slightly more involved, because (a) it has to be checked that one can always perform this perturbation consistently across different $h$, (b) one needs to perform this perturbation in a measurable fashion across bids and types so that the composition of the original distributional strategy and the perturbation remains a valid distributional strategy, and (c) the possibility that odd tie-breaking might result in a small change in a nonmarginal bid affecting whether or not a marginal bid wins must be considered.

To see Lemma 8, assume that there is a positive probability of a competitive tie or a noncompetitive but non-trade-maximizing tie at $b^{*}$. Since $m$ is undominated*, by Lemma 5, a player will not submit a buy bid $b_{i h}=b^{*}$ where $v_{i h}<p_{i h e_{i}}\left(b_{i h}\right)$ or a sell bid where $v_{i h}>p_{i h e_{i}}\left(b_{i h}\right)$. Since the distribution of values is atomless, there is zero probability that $v_{i h}=p_{i h e_{i}}\left(b^{*}\right)$. Hence, almost all the buyers at that tie would strictly prefer to buy, and almost all sellers would prefer to sell. But, no matter what the omniscient tie-breaking rule, if there is a positive probability of a competitive tie or a noncompetitive and non-trademaximizing tie at some $b^{*}$, then at least one player who would benefit from trade is sometimes "losing" the tie, and so strictly benefits by the deviation described above.

When we put Lemmas 7 and 8 together, we end up with the following implication.

THEOREM 9 (Invariance): If an auction $\left(P, o, t, b_{0}\right)$ satisfies Assumptions $1-10$, and an undominated ${ }^{*}$ profile of distributional strategies $m$ is an equilibrium, then under $m$ there is zero probability of a competitive tie or noncompetitive ties where $o$ is not trade-maximizing, and $m$ remains an equilibrium for $\left(P, o^{\prime}, t, b_{0}\right)$ for any trade-maximizing tie-breaking rule $o^{\prime}$. 
Note that the conclusion that we can switch from $o$ to $o^{\prime}$ and still have $m$ be an equilibrium, is not a direct implication of Lemma 8. It may be that $m$ is an equilibrium under $o$, but $o^{\prime}$ would induce some player to deviate to establish a new tie. This possibility is ruled out using Lemma 7, as the following short proof shows.

Proof of TheOREM 9: The fact that $m$ must be free of competitive ties and noncompetitive ties where $o$ is not trade-maximizing follows directly from Lemma 8. Let us argue that $m$ is also an equilibrium for $\left(P, o^{\prime}, t, b_{0}\right)$. Given that any ties occurring with positive probability under $m$ must be noncompetitive and where $o$ is trade-maximizing, $m$ must lead to the same payoff vector $u$ under both $o$ and $o^{\prime}$. Now suppose to the contrary of the theorem that $m$ is not an equilibrium under $o^{\prime}$. Then there exists $i$ and $m_{i}^{\prime}$ such that $\pi_{i}\left(m_{-i}, m_{i}^{\prime}, P, o^{\prime}, t, b_{0}\right)>u_{i}$. By Lemma 7 we can find $m_{i}^{\prime \prime}$ which is tie-free for $i$ and such that $\pi_{i}\left(m_{-i}, m_{i}^{\prime \prime}, P, o^{\prime}, t, b_{0}\right)>u_{i}$. Since $m_{-i}, m_{i}^{\prime \prime}$ is tie-free for $i$ it follows that $\pi_{i}\left(m_{-i}, m_{i}^{\prime \prime}, P, o, t, b_{0}\right)=\pi_{i}\left(m_{-i}, m_{i}^{\prime \prime}, P, o^{\prime}, t, b_{0}\right)>u_{i}$, contradicting the fact that $m$ is an equilibrium at $o$.

Q.E.D.

The conclusions of Theorem 9 can fail if one ventures beyond private values. This can be seen in Example 1 of JSSZ, where there exists an equilibrium for a nonstandard tie-breaking rule, but none exists for standard tie-breaking.

Theorem 9 establishes that any undominated* equilibrium can only involve trade-maximizing noncompetitive ties, and will remain an equilibrium for any trade-maximizing tie-breaking rule. Thus, to prove Theorem 6 we need only prove that there exists an undominated* equilibrium for some tie-breaking rule.

We think that it is instructive to offer proofs via both JSSZ and Reny, as at this point they are both fairly straightforward. Moreover, this clarifies the relationship between these two methodologies, which may be useful in further applications and in understanding existence issues more broadly.

\subsection{Two Proofs of Theorem 3}

We first construct an auxiliary game where dominated strategies are penalized according to their distance from the set of undominated strategies. Equilibria in this game must involve undominated strategies. It is also easy to see that these remain equilibria when the penalty of domination is removed (being careful with some details regarding the tie-breaking rule). This is stated in the following lemma.

Consider the game $G\left(P, o, t, b_{0}\right)$ in which when any player $i$ uses $b_{i}$ with type $e_{i}, v_{i}$, he pays a penalty $c_{i}\left(e_{i}, v_{i}, b_{i}\right)$ in additional to the payoff he receives from $\left(P, o, t, b_{0}\right)$, where $c_{i}$ is the distance of a point from the set $W_{i}{ }^{33}$

\footnotetext{
${ }^{33}$ Take the distance of a point from the (closed) set $W_{i}$ to be the minimum of the distances from that point to points in the set.
} 
Lemma 10: Consider an auction $\left(P, o, t, b_{0}\right)$ and the auxiliary $G\left(P, o, t, b_{0}\right)$ that penalizes weakly dominated strategies. Let $m$ be an equilibrium of $G(P, o$, $\left.t, b_{0}\right)$. Then, $m$ is undominated ${ }^{*}$ and is an equilibrium of the original auction $\left(P, o, t, b_{0}\right)$.

If $G\left(P, o, t, b_{0}\right)$ has an equilibrium $m$ for some $o$, then by Lemma 10 , so does the original auction. Then by Theorem $9, m$ remains an equilibrium for any other trade-maximizing $o$. This would then complete the proof of Theorem 6 .

\section{Proving Theorem 6 using JSSZ's endogenous tie-breaking rules}

Theorem 1 in JSSZ implies that there exists an equilibrium $m$ in an augmented form of $G\left(P, o, t, b_{0}\right)$ where players also (truthfully) announce their types and tie-breaking depends on those announcements. Those strategies remain an equilibrium when we ignore type announcements, and change the tie-breaking rule to directly depend on types rather than announced types. Thus, there exists an equilibrium of $G\left(P, o, t, b_{0}\right)$ and hence the original auction for some omniscient tie-breaking rule $o$, and we are done.

The only detail to check is that $G\left(P, o, t, b_{0}\right)$ falls into the class of games identified by JSSZ. This follows from checking two things. First, it is clear that the correspondence taking bid vectors into distributions over consistent allocations (outcomes in JSSZ) is upper hemi-continuous, nonempty, and convex-valued. Second, JSSZ write payoffs as an inner product of a finite set of functions $u_{k}$ that are continuous in actions and types, and an outcome function that depends on actions and leads to probabilities over the $u_{k}$ 's. Here, the $u_{k}$ 's are simply the utilities that a player gets conditional on getting a fixed number of objects, and the outcome function is the tie-breaking rule (see (1)). These are continuous, given the continuity both of the payment rules $t$, and of the penalty to weakly dominated strategies.

\section{Proving Theorem 6 using Reny's better-reply-security}

Again, we consider $G\left(P, o, t, b_{0}\right)$. In fact, here the penalties are necessary not only for establishing existence of an equilibrium in non-weakly dominated strategies, but because the game without these penalties can fail to satisfy the conditions of Reny. We return to illustrate this with an example below.

The following definitions and conditions from Reny are the critical ones.

A player $i$ can secure a payoff of $\alpha$ at $m_{-i}$ if there is a strategy $m_{i}$ such that $\pi_{i}\left(m_{-i}^{\prime}, m_{i}\right) \geq \alpha$ for all $m_{-i}^{\prime}$ in some neighborhood of $m_{-i}{ }^{34}$

A game is better reply secure if for any $m^{*}, u^{*}$ in the closure of the graph of the vector payoff function, if $m^{*}$ is not an equilibrium, then there exists $i$ who can secure a payoff strictly above $u_{i}^{*}$ at $m_{-i}^{*}$.

\footnotetext{
${ }^{34}$ Again, use the topology of weak convergence of measures on the distributional strategies (which leads to a compact set of strategies).
} 
It follows from Theorem 3.1 in Reny (1999) that if $G\left(P, o, t, b_{0}\right)$ is better reply secure, then $G\left(P, o, t, b_{0}\right)$ has an equilibrium, and we will have once again concluded the proof of Theorem $6 .{ }^{35}$

So, it remains to verify that the (penalized) auction satisfies better-replysecurity. It is tempting to read better-reply-security as saying "whenever a player has a better reply, he has a secure better reply" and downplay the importance of taking the closure of the graph of the payoff function of the game rather than just considering the graph itself. The following example shows the key role played by the closure operation.

EXAMPLE 11: Consider a first price auction (with standard tie-breaking) with two players and one object for sale. Player 1's value is always 1 and player 2's value is always 2 . This game violates better-reply-security. In particular, consider $m^{*}, u^{*}$ where both players bid 1 and $u^{*}=(0,1)$ (because player 2 always wins the object). This is the limit, for instance, of a sequence of bids where player 1 always bids 1 and 2's bids approach 1 from above. ${ }^{36}$ It is easy to see that $m^{*}$ is not an equilibrium of the auction and yet neither player can secure (or even obtain) a payoff above $u^{*}$ via a deviation. Hence better-replysecurity fails. If one just considers $m, u$ 's in the graph of the game, then it is easy to check that for any better reply there exists a secure better reply. So, the failure of better-reply-security comes at a point in the closure of the graph of the game, but not in the graph of the game.

Fascinatingly, despite the fact that this game fails better-reply-security, not only does it have Nash equilibria, but $m^{*}, u^{*}$ is in fact the limit of such equilibria! Let player 1 randomize uniformly over a small interval $(1-\varepsilon, 1)$, and player 2 always bid $1 .{ }^{37}$ Player 1 does not want to bid more, since the object is only worth 1 to him. And, for small enough $\varepsilon$, player 2 does not want to bid anything less than 1 . As $\varepsilon \rightarrow 0$, the limit distributional strategies and payoffs are $m^{*}, u^{*}$. This provides a sharp example that shows that better-reply-security is sufficient but not necessary for existence.

It is also useful to point out that if one breaks all ties in favor of player 2, then $m^{*}, u^{*}$ is Nash. This illustrates that our invariance result (Theorem 9) can fail when there are atoms in the distribution of types.

The example makes it clear that to verify better-reply-security we need to have some handle on the possible $m^{*}, u^{*}$ that are in the closure of the graph

\footnotetext{
${ }^{35}$ Reny's Theorem 3.1 requires a quasi-concavity of payoffs and a compactness of strategies. Compactness has already been established. Even though the $U_{i}$ 's we have considered here are not necessarily quasi-concave, note that payoffs are in fact linear in $m$, as these are distributional strategies and preferences are von Neumann-Morgenstern.

${ }^{36}$ It is useful to point out that endogenous tie-breaking would actually prescribe the tiebreaking rule that gives the object to player 2 at a tied bid of 1 , and would lead to an equilibrium under this alternative tie-breaking rule.

${ }^{37}$ This type of equilibrium was identified in the context of asymmetric Bertrand games by Blume (2003).
} 
of the auction in question. Generally, doing this directly will be complicated. For instance, consider a bid vector with three tied players 1, 2, 3; one could approach this vector from a sequence that has player 3 always winning, or with 1 and 2 tied and always winning, or any of a continuum of other possibilities. Once we are in auctions with many objects and allow for the mixing possible under distributional strategies, the potential limit points are quite complex. ${ }^{38}$

The key to verifying better-reply-security comes back to the use of omniscient tie-breaking rules! This is captured in the following lemma.

LEMma 12: Consider an auction $\left(P, o, t, b_{0}\right)$. For each $m^{*}, u^{*}$ in the closure of the graph $G\left(P, o, t, b_{0}\right)$, there exists an omniscient tie-breaking rule $o^{*}$ such that $u^{*}$ is the payoff vector induced under $G\left(P, o^{*}, t, b_{0}\right)$ by $m^{*}$.

Proof OF LEMMA 12: Consider a sequence $\left(m^{k}, u^{k}\right)$ in the graph $G(P, o$, $t, b_{0}$ ) converging to $m^{*}, u^{*}$. Then each $m^{k}$ along with $o$ induces a measure $M^{k}$ on $\Theta \times \mathcal{B} \times A$, which leads to the utilities $u^{k}$. Taking a subsequence if necessary, the sequence of $M^{k}$ 's converges weakly to a measure $M^{*}$ on $\Theta \times \mathcal{B} \times A$. Given that utility functions are continuous on $\Theta \times \mathcal{B} \times A$ ( $A$ is finite), the corresponding utilities induced under $M^{*}$ are $u^{*}$. Note that $M^{*}$ can be induced by an omniscient tie-breaking rule $o^{*}$ coupled with $m^{*}$ (see, for instance, Lemma 2 in Simon and Zame (1990)).

Q.E.D.

Now let us use Lemma 12 to verify better-reply-security to complete the proof of Theorem 6 .

Consider any $m^{*}, u^{*}$ in the closure of the graph of $G\left(P, o, t, b_{0}\right)$ which is not an equilibrium of $G\left(P, o, t, b_{0}\right)$. By Lemma 12 there exists an omniscient tie-breaking rule $o^{*}$ such that $u^{*}$ is the payoff vector induced under $G\left(P, o^{*}, t, b_{0}\right)$ by $m^{*}$. By Lemma 10 we can deduce that $m^{*}$ is not an equilibrium of $G\left(P, o^{*}, t, b_{0}\right)$. To see this, suppose otherwise. Then by Lemma $10 \mathrm{~m}^{*}$ is an equilibrium of $\left(P, o^{*}, t, b_{0}\right)$ and is undominated*. Then by Theorem 9 it would be an equilibrium of $\left(P, o, t, b_{0}\right)$ and since $m^{*}$ is undominated ${ }^{*}$, it would

\footnotetext{
${ }^{38}$ Recall from the Introduction that another way to apply Reny's result is via reciprocal upper semicontinuity, and tie-breaking that maximizes the sum of the player surpluses. Under this technique, one can a priori restrict agents to the closure of the nonweakly dominated strategies, side-stepping the penalty functions we use. Reciprocal upper semicontinuity is intuitively obvious, since any ties that exist along a sequence of bid profiles also exist in the limit, and hence the auctioneer has at least as much discretion in the limit as he did late in the sequence. Because we are working in the space of distributional strategies, this pointwise argument needs to be applied with some care. One also has to be careful about the difference between efficient tie-breaking and that which maximizes the sum of payoffs to players other than 0 . Reciprocal upper semicontinuity can fail under efficient tie-breaking (for instance, it is easy to generate examples where along a sequence, allocations are inefficient, but result in low payments to the auctioneer, while the limiting efficient allocation raises these payments by enough so that players are worse off in aggregate). We chose the direct application of Reny's better-reply-security because of the insight it provides into the relationship between better-reply-security and endogenous tie-breaking rules.
} 
also be an equilibrium of $G\left(P, o, t, b_{0}\right)$, which would be a contradiction. Thus there exists an improving deviation $m_{i}$ relative to the game $G\left(P, o^{*}, t, b_{0}\right)$. By Lemma 7, this improving deviation $m_{i}$ can be taken to be tie-free. ${ }^{39}$ Then $m_{i}$ remains tie-free for all strategies in a neighborhood of $m_{-i}^{*}$, and so player $i$ 's payoff is continuous in $m_{-i}$ in this neighborhood and the payoff is independent of the tie-breaking rule. Thus, $m_{i}$ secures a payoff above $u_{i}^{*}$ at $m_{-i}^{*}$ relative to $G\left(P, o, t, b_{0}\right)$, as required by better-reply-security.

Finally, let us return to the issue of why penalizing weakly dominated strategies is important to verifying better-reply-security.

EXAMPLE 13: Consider a two player and one object first price auction, with standard tie-breaking. Player 1 has $v_{1}$ uniformly distributed on $[0,1]$, and player 2 has $v_{2}$ uniformly distributed on $[2,3]$. This auction satisfies all of our assumptions, and indeed has an equilibrium. Nevertheless, $\left(P, o^{*}, t, b_{0}\right)$ fails to satisfy better-reply-security; only $G\left(P, o^{*}, t, b_{0}\right)$ satisfies it. To see this, consider $m^{*}, u^{*}$ such that under $m^{*}$ both players always bid 1.5 and $u^{*}=(0,1)$. This is in the closure of the graph, by considering a sequence where player 1 always bids 1.5 and player 2 bids $1.5+(1 / k)$ (regardless of valuation). Relative to $u^{*}$, neither player has an improving deviation, even though $m^{*}$ is not an equilibrium of the auction with the usual tie-breaking rule, and so better-replysecurity fails.

\subsection{Comments on Endogenous Sharing and Better-Reply-Security}

It is interesting that the tricky part of the proof using better-reply-security is to get a handle on the $u^{*}$ 's in the closure of the game. The fact that they are those generated by omniscient tie-breaking suggests a deeper connection between the machinery of Reny and that of JSSZ. That is, a proof of existence via "apply JSSZ and check that some equilibria correspond to nice tie-breaking" and "check better-reply-security" are closely related. Because of the requirement that better-reply-security apply relative to all points in the closure of the graph, rather than just the graph, one has to understand exactly what might be in that closure; and the points in the closure are precisely the points that come from omniscient choices at points of discontinuity. On the other hand, in applying JSSZ, one has to understand the equilibria that might be generated under omniscient choices at points of discontinuity. In the auction setting, these two tasks are closely related. How these approaches turn out to be related and which might be more efficient in other settings is an open question.

\footnotetext{
${ }^{39}$ To be careful, Lemma 7 would apply to $G\left(P, o^{*}, t, b_{0}\right)$ rather than $\left(P, o^{*}, t, b_{0}\right)$. It is a direct extension that it holds for $G\left(P, o^{*}, t, b_{0}\right)$ as well.
} 


\subsection{A Corollary on Monotone Pure Strategy Equilibria}

As mentioned in the Introduction, while Theorem 6 establishes existence of equilibrium in distributional strategies, in some contexts one can use additional structure to argue for the existence of monotone pure strategy equilibria. ${ }^{40}$ Let us present one such result.

Consider a situation where the distribution over types is independent across players and players are risk-neutral.

In order to establish this result for this general class of auctions, we need to impose some restrictions on the interaction of a player's bids for different objects. We strengthen (Assumption 8) as follows.

For any $(e, b)$ and $i$ such that $i$ is not involved in any competitive ties (so that $i$ 's allocation is unique), let $T_{i}(e, b)$ be the total payment made by $i$.

AsSUMPTION 8': Assumption 8 holds. In addition, consider any $(e, b)$ and $\left(e, b_{-i}, b_{i}^{\prime}\right)$ such that $i$ is not involved in any competitive ties, and $h$ such that $b_{i,-h}, b_{i h}^{\prime}$ and $b_{i,-h}^{\prime}, b_{i h}$ are both valid bid vectors. Then,

$$
\begin{aligned}
& T\left(e, b_{-i}, b_{i}\right)-T\left(e, b_{-i}, b_{i,-h}, b_{i h}^{\prime}\right) \\
& \quad=T\left(e, b_{-i}, b_{i,-h}^{\prime}, b_{i h}\right)-T\left(e, b_{-i}, b_{i}^{\prime}\right) .
\end{aligned}
$$

Assumption $8^{\prime}$ states that, holding the overall endowment and the bids of the other players constant, the effect of a change in $i$ 's $h$ th bid on his payments is a function only of what the change in that $h$ th bid is, irrespective of $i$ 's other bids as long as this change is feasible. ${ }^{41}$ This condition is satisfied by all the standard auction formats. In (multi-unit) discriminatory and all pay auctions a change in $i$ 's $h$ th bid only affects the payment attributed to the $h$ th object (in the all pay auction this is whether or not the object is won), and so the change in payment is unaffected by the rest of $i$ 's bid vector. In a Vickrey auction, the change in $i$ 's $h$ th bid can only affect whether $i$ gets an $h$ th object, and if it does, then the price for that additional object depends on the bids of the other agents, and is independent of the rest of $i$ 's bid vector. In a multi-unit uniform price auction, an additional feature arises in that the $h$ th bid might be setting the price for $i$ 's other objects. However, for any feasible bid vector where $i$ 's $h$ th bid is setting the price, $i$ must be getting $h-1$ objects, and so the change in payments is then

\footnotetext{
${ }^{40}$ In terms of distributional strategies, a pure strategy is any strategy that puts probability one on the graph of a function from types to bids, $f: \Theta \rightarrow \mathcal{B}$, where $f=\left(f_{1}, \ldots, f_{n}\right)$ and $f_{i}: \Theta_{i} \rightarrow \mathcal{B}_{i}$. A strategy is monotone if the $f$ is such that higher valuation vectors lead to (weakly) higher bid vectors-holding the endowment constant. That is, for any $i$, if $v_{i h}^{\prime}>v_{i h}$ for each $h$, then $f_{i}\left(v_{i}^{\prime}, e_{i}\right) \geq$ $f\left(v_{i}, e_{i}\right)$.

${ }^{41}$ Given Assumption 8, a sufficient condition for Assumption $8^{\prime}$ is that $\partial T_{i}(e, b) / \partial b_{i h}$ be well defined when no other bidder has a bid equal to $b_{i h}$, and this partial derivative depends only on $(h, e), b_{i h}$, and the rank of $b_{i h}$ among all bids.
} 
also irrespective of the level of $i$ 's other bids subject to this change of the $h$ th bid being feasible.

This rules out settings where, for example, the price to players other than some player $j$ in a multiple unit auction is a smoothly changing convex combination of the Vickrey and highest rejected bid rule, depending on the bid of player $j$. It also rules out a multiple unit variant of the third price auction in which the price is set by the second highest rejected bid. In this setting, knowledge that one's second bid has rank $\sum e_{i}+2$ does not tie down the effect of a change in bid on payments, since one's highest bid may or may not be among the first $\sum e_{i}$.

COROLlary 14: Consider any auction satisfying Assumptions 1-10 and Assumption 8', where types are independent across players, the marginal distribution of each player's vector of values is absolutely continuous with respect to $\ell$-dimensional Lebesgue measure, and each player $i \geq 1$ is risk-neutral. There exists a monotone pure strategy equilibrium in undominated* ${ }^{*}$ strategies with a zero probability of competitive ties, which is an equilibrium under any omniscient and effectively trade-maximizing tie-breaking rule, including standard tie-breaking.

The proof of Corollary 14 appears in the Appendix. The basic ideas are fairly straightforward, although the details are slightly more involved. Under Assumption 8', risk-neutrality, and independence, a change in a bid for the $h$ th object by $i$ can be considered essentially independently of bids for other objects. So, a change in one bid does not change the effect on payments of a change in another. And, independence also implies that the change of a bid affects payments and the probability of winning a given object in a way that is independent of a player's valuation. Thus, given private values, if raising a bid is weakly beneficial at one valuation, it is strictly beneficial at any higher valuation. This implies monotonicity and pure strategies to the extent that changes in bids matter. To complete the picture, we use a modification of the auction that is similar to the seeding argument we use to prove positive trade in the next section, and is also in the spirit of a technique subsequently used by Kazumori (2003) to argue for existence of monotone pure strategy equilibria. In particular, with a small probability one introduces extra bidders who randomly bid at all levels for all objects and vary the available supply to take on any possible value. This implies that any change in bids matters, and then bids must be monotone and hence pure (almost) everywhere. Taking careful limits as the noise goes to 0 , one can use results of JSSZ and the invariance results (Theorem 9) to deduce a monotone pure strategy equilibrium of the original auction.

It is also worth noting that even without the perturbation arguments, bids must be increasing over ranges where they matter. What is possible is that some equilibria might involve players mixing or bidding nonmonotonically over a range of bids all of which, for example, never win. In the single unit case, one can effectively purify such equilibria. Doing so in the multiple unit case seems 
more daunting, and so it is open whether the auctions described might have other equilibria meaningfully different from the monotone equilibria we have shown to exist.

\section{EQUILIBRIA WITH A POSITIVE PROBABILITY OF TRADE}

For some auction settings, a major potential weakness in the existence result (Theorem 6) as currently stated is that there may be a zero probability of trade. Consider, for example, a simple double auction (as in Example 9). There is an equilibrium that puts probability one on the closure of the set of undominated strategies in this auction where sellers always bid $\bar{v}$, and buyers always bid 0 . Buyers know that sellers are never making serious bids and sellers know that buyers are never making serious bids, and thus their own nonserious bids are best responses, and yet undominated strategies are used..$^{42}$ So, it could be that Theorem 6 has only "proven" that this degenerate equilibrium exists.

In this section we establish the existence of an equilibrium with a positive probability of trade for the auctions in our class.

First, we note that in some cases it is straightforward to see that each equilibrium identified in Theorem 6 must involve a positive probability of trade. In particular, consider the case where the nonstrategic player 0 is active in the sense that $P_{0}\left(\left\{e_{0}>0\right\}\right)>0$ and that when $e_{0}>0$, player 0 sets a reserve price for at least one of his units so that there is a positive probability that some buyer has a value for a first unit that would lead to a positive utility if they were to be allocated it at a bid of the reserve price (for simple pricing rules, this reduces simply to a reserve below the highest possible buy value). Then, there is clearly trade in equilibrium.

So, the issue of establishing existence of equilibria with a positive probability of trade involves settings where player 0 is not active, as in a double auction. We use three additional assumptions. The first two are critical, the third a convenience.

The first assumption rules out too much dependence in the distribution of values. Essentially, it requires only that changing one player's type does not alter the support of possible types for another, and is consistent with any finite likelihood ratio on the distribution of values within that support. As such, it is quite weak.

Assumption 11 (Full Support Prior): $f(\theta)>0$ for all $\theta \in \Theta$, where $f$ is the Radon-Nikodym derivative of $P$ with respect to $\prod_{i} P_{i}$.

Given that $f$ is a continuous function (Assumption 3) and $\Theta$ is compact (Assumption 2), $f(\theta)>0$ implies that there exists $\infty>M>M^{\prime}>0$ such that

\footnotetext{
${ }^{42}$ In fact, the equilibrium satisfies a trembling hand perfection requirement, as discussed in Jackson and Swinkels (1999).
} 
$M>f(\theta)>M^{\prime}$ for all $\theta \in \Theta$. The new content of Assumption 11 over that of Assumptions 2 and 3, is of course, the existence of $M^{\prime}{ }^{43}$

We use Assumption 11 to show that the fact that types are not perfectly correlated translates into the same feature for bids (see Lemma 17 in the Appendix).

Our second assumption ensures that there is some possibility of serious competition for the gains to trade. This guarantees that some players (e.g., buyers) will be forced to make bids that are attractive enough to induce other players (e.g., sellers) to be active.

AsSUMPTION 12 (Competition for Gains from Trade): Let $\bar{w}$ be the max of the support of the distribution over all buy values, and $\underline{w}$ the min of the support of the distribution over sell values. Let $[\underline{w}, \bar{w}] \subset[\underline{b}, \bar{b}]$ and at least one of the following hold:

(i) For each player who ever has a buy value above $\underline{w}$, there is a positive probability that the remaining players have at least $\ell+1$ sell values below $\bar{w}$, or

(ii) for each player who ever has a sell value below $\bar{w}$, there is a positive probability that the remaining players have at least $\ell+1$ buy values above $\underline{w}$.

For some insight into Assumption 12, consider a standard double auction. Then, as long as there are two or more sellers who sometimes have values below $\bar{w}$ or two or more buyers who sometimes have values above $\underline{w}$, Assumption 12 is satisfied. Within the standard double auction, we are ruling out only the case of one buyer and one seller. ${ }^{44}$ The condition involving $\ell+1$ extends this to multiple unit demands. It rules out, for example, a setting in which there is a single buyer demanding three units, and two sellers, each with one unit to sell. Here, there is no real competition among the sellers. This assumption of having competition is used in our proof of Theorem 15 in showing that seeding trade then leads to positive amounts of trade, but this condition is clearly not always necessary for getting positive trade in equilibrium.

When players can be either buyers or sellers a priori, the condition is slightly more intricate. In particular, consider a case where there are three players. Player 1 always has endowment 0 , and value uniform on [.5, .6]. Player 2 always has endowment 1 , and value uniform on $[.4, .5]$. Finally, player 3 half the time has endowment 0 and value uniform on $[.9,1]$, and half the time endowment 1 and value uniform on $[0, .1]$. Then, the condition fails, because the only time that player 3 has a high buy value, there is only one seller, and the only time player 3 has a low sell value, there is only one buyer. Hence, there is really no competition for the gains to trade generated by the existence of player 3 .

\footnotetext{
${ }^{43}$ It should be noted that the amount of trade guaranteed by the proof of Theorem 15 depends on $M^{\prime}$, and that when $M^{\prime}$ is small, so is the amount of trade that is guaranteed to occur. See Cripps and Swinkels (2003) for a demonstration that under mild conditions, as the number of players grows, trade in any such nontrivial equilibria converges to the Walrasian level.

${ }^{44}$ See Kadan (2004) for a proof of existence in the bilateral case.
} 
Finally, in establishing existence, we did not need a direct tie between the level of bids and the prices paid, other than some continuity and monotonicity conditions. In order to use a "seeding trade" argument, it is convenient to have a tighter feel for how payments vary with bids. The following assumption does this.

Assumption 13 (Marginal Transfers Equal Bids): For any $i, h$, and $e$, $t_{i}(h, e, b)-t_{i}(h-1, e, b)=b_{i h}$.

Assumption 13 states that when a player is involved in a tie, he pays his bid. This is true for any sensible double auction rule, as when there is a tie at $b^{*}$, then $b^{*}$ is the only price which clears the market for the submitted demand and supply curves.

We can now state our second main result.

THEOREM 15: Each auction ( $\left.P, o, t, b_{0}\right)$ satisfying Assumptions 1-13 has an undominated ${ }^{*}$ equilibrium that has a positive probability of trade.

Given Theorem 15, and recalling our discussion of auctions in which player 0 is active, we obtain the following corollary.

COROLlaRY 16: Let $\left(P, o, t, b_{0}\right)$ satisfy Assumptions 1-10. If either player 0 is active $^{45}$ (as in a one-sided auction), or Assumptions 11-13 are satisfied, then there exists an undominated ${ }^{*}$ equilibrium that has a positive probability of trade.

The idea of the proof of Theorem 15 can be seen in a simplified setting. Think about the case of two buyers, each desiring one object, and two sellers, each with one object to sell, where the marginal of $P$ onto each player's value has support $[\underline{w}, \bar{w}]$, and where values are not too dependent, so that Assumption 11 is satisfied. Since buyers and sellers are distinct, Assumption 12 is also satisfied.

We "seed" trade in the following manner. Consider a sequence of modified auctions indexed by $x$, in which we add a fifth player who $1 / x$ of the time has $e_{5}=1$ and makes a sell offer which is uniform on $[\underline{w}, \bar{w}], 1 / x$ of the time has $e_{5}=0$ and makes a buy offer which is uniform on $[\underline{w}, \bar{w}]$, and the remainder of the time has $e_{5}=0$ and makes no bids, where all of this is independent of $(e, v)$. Clearly, any equilibrium in auction $x$ must have some trade, as otherwise someone should behave in a way to trade with player 5 when he is active.

Now let us argue that trade is in fact bounded away from zero as $x \rightarrow \infty$. Suppose to the contrary that as $x \rightarrow \infty$, the amount of trade heads to zero. The only way this can happen is that the probability that buyers offer above

\footnotetext{
${ }^{45}$ This requires that player 0 set a reserve price for selling at least one of the objects below $\bar{w}$ (or above $\underline{w}$ in a procurement auction).
} 
$\underline{w}$ vanishes as does the probability that sellers ask less than $\bar{w}$. To see this, assume for example, that there is probability $\gamma$ of a sell bid below $\bar{w}-\gamma$. Then this sell bid must go unfilled with a probability going to one as otherwise there would be a nondegenerate amount of trade. So, consider the strategy for buyers where they bid $\bar{w}-\gamma$ whenever they have a value of at least $\bar{w}-\gamma / 2$. Under Assumption 11 this would win with a probability bounded away from 0 . Hence, there is a strategy available to buyers that earns an amount bounded away from zero. But then, equilibrium profits must also be bounded away from zero, which is inconsistent with the expected amount of trade going to zero.

So, we have argued that in order for trade to vanish, it must be that with probability approaching one both sellers are asking almost $\bar{w}$, and both buyers are offering nearly $\underline{w}$. However, this cannot be part of an equilibrium by the following argument. Because of Assumption 12, there is a positive probability that simultaneously, both sellers (or both buyers) would be willing to buy the object at the value of one of the buyers. So, think about deviating to a slightly lower bid as a seller. If there was no high buy bid, this is costless. So, we can condition payoffs on the event that there is at least one high buy bid, whether by player 5 or by one of the buyers. Because of player 5 , this is a positive probability event. But, for each of player 5 and the two buyers, the probability of a high buy bid is very small. So, since values (and hence bids) are somewhat independent, conditional on there being any high buy bid, there is almost surely only one high buy bid. But then, since there is a positive probability of both sellers simultaneously being interested, they will have an incentive to compete against each other to be the one who trades in this event. This rules out that sell bids are very high almost always, giving a contradiction.

Essentially then, the presence of the extra player pushes the equilibrium away from the pure no-trade one. But, once there is some trade, Assumption 12 implies that there will be competition by sellers (or buyers) to be the one who trades in the rare event that it occurs. This competition pushes bids away from the boundary in a way that implies a minimum positive level of trade that is independent of $x$. In the limit, as $x \rightarrow \infty$ this generates a positive trade equilibrium of the original game.

Matthew O. Jackson, Humanities and Social Sciences 228-77, California Institute of Technology, Pasadena, CA 91125, U.S.A.; jacksonm@hss.caltech.edu and

Jeroen M. Swinkels, Olin School of Business, Washington University in St. Louis, St. Louis, MO 63130, U.S.A.; swinkels@wustl.edu.

Manuscript received November, 2001; final revision received April, 2004.

\section{APPENDIX}

Proof of Lemma 5: Assume $b$ is such that $p_{i h e_{i}}\left(b_{i h}\right)<v_{i h}$ for some $h \leq e_{i}$. Let $h^{\prime}$ be the lowest index for which this is true. Now, by definition, $b_{i, h^{\prime}-1} \geq b_{i, h^{\prime}}$. And, since 
$p_{i, h^{\prime}-1, e_{i}}\left(b_{i, h^{\prime}-1}\right) \geq v_{i, h^{\prime}-1} \geq v_{i, h^{\prime}}>p_{i, h^{\prime}, e_{i}}\left(b_{i, h^{\prime}}\right)$, it follows that $b_{i, h^{\prime}-1}>b_{i, h^{\prime}}$ (recall from Assumption 9 that $p_{i, h^{\prime}-1, e_{i}}(x) \leq p_{i, h^{\prime}, e_{i}}(x)$, so it cannot be the case that $\left.b_{i, h^{\prime}-1}=b_{i, h}\right)$. Raise $b_{i h^{\prime}}$ by $\varepsilon>0$, where $\varepsilon<b_{i, h^{\prime}-1}-b_{i, h^{\prime}}$, and where $v_{i, h^{\prime}}>p_{i, h^{\prime}, e_{i}}\left(b_{i h^{\prime}}^{\prime}\right)$ for all $b_{i h^{\prime}}^{\prime} \in\left[b_{i h^{\prime}}, b_{i h^{\prime}}+\varepsilon\right]$. Since $p_{i, h^{\prime}, e_{i}}(\cdot)$ is continuous (by the continuity of the $t_{i}$ 's), such an $\varepsilon$ exists.

Now, for any given $b_{-i}, e_{-i}$, let $b^{*}$ be the $\sum e_{i}-h^{\prime}$ highest bid submitted by players other than $i$. Consider raising $i$ 's $h^{\prime}$ th bid continuously from $b_{i h^{\prime}}$ to $b_{i h^{\prime}}+\varepsilon$. As long as the allocation is unchanging (i.e., except at $b^{*}$, if $b^{*}$ should happen to be in $\left[b_{i h^{\prime}}, b_{i h^{\prime}}+\varepsilon\right]$ ), this is irrelevant if $i$ is currently a net buyer (is receiving $e_{i}$ or more objects), and either irrelevant or helpful if $i$ is currently a net seller (receiving strictly less than $e_{i}$ objects), in each case using Assumption 7. So, consider what happens as one goes from just below $b^{*}$ to $b^{*}$, or from $b^{*}$ to just above $b^{*}$. In each case, either the allocation is unchanged (if tie-breaking at $b^{*}$ happens to be fortuitous), or $i$ 's allocation goes up by one unit (that is, he sells one less object). But, $v_{i, h^{\prime}}>p_{i, h^{\prime}, e_{i}}\left(b^{*}\right)$ by choice of $\varepsilon$. Hence, such a change will strictly improve $i$ 's payoff. And, for $b^{*} \in\left(b_{i h^{\prime}}, b_{i h^{\prime}}+\varepsilon\right)$ such a change in allocation must occur. It follows that the original bid vector $b$ is weakly dominated by $b^{\prime}$ defined in this way.

But, notice that if one chooses any $\left(e_{i}^{\prime}, v_{i}^{\prime}, b_{i}^{\prime}\right)$ sufficiently close to $\left(e_{i}, v_{i}, b_{i}\right)$, it will remain the case that $p_{i h e_{i}^{\prime}}\left(b_{i h}^{\prime}\right)<v_{i h}^{\prime}$ and so $\left(e_{i}, v_{i}, b_{i}\right) \notin W_{i}^{\prime}$ (note that $e_{i}^{\prime}$ must equal $e_{i}$ for close by $\left(e_{i}^{\prime}, v_{i}^{\prime}, b_{i}^{\prime}\right)$ ). Hence, there is a ball around $\left(e_{i}, v_{i}, b_{i}\right)$ with empty intersection with $W_{i}^{\prime}$, and $\left(e_{i}, v_{i}, b_{i}\right) \notin W_{i}$. The proof for $h>e_{i}$ is the same.

Q.E.D.

The following lemmas are useful in the proofs of Lemmas 7 and 8 and Theorem 15.

Fix a profile of strategies $\left(m_{1}^{\prime}, \ldots, m_{n}^{\prime}\right)$ (equilibrium or otherwise). Given these strategies, let $B$ be the induced measure over $\mathcal{B} \times \Theta$. Let $B_{i}$ be the marginal of $B$ on $\mathcal{B}_{i} \times \Theta_{i}$ for $i \in N$ and let $B_{0}$ be simply $P_{0}$, the marginal of $P$ onto $e_{0}$.

Our next lemma shows that an implication of the absolute continuity of $P$ with respect to $\prod_{i=0}^{n} P_{i}$ (Assumption 3 ) is that $B$ is absolutely continuous with respect to $\prod_{i=0}^{n} B_{i}$. So, events involving the set of submitted bids and realized values that are zero probability assuming players draw values and bid independently are also zero probability under the actual distribution over realized values and submitted bids. If, in addition, $\prod_{i=0}^{n} P_{i}$ is absolutely continuous with respect to $P$ (Assumption 11), then the reverse implication will be true as well, so that positive probability events under $\prod_{i=0}^{n} B_{i}$ are positive probability under $B$.

LEMMA 17: (i) Under Assumptions 2-4, for each strategy profile $m^{\prime}, B$ is absolutely continuous with respect to $\prod_{i=0}^{n} B_{i}$. (ii) If Assumption 11 is also satisfied, then $\prod_{i=0}^{n} B_{i}$ is absolutely continuous with respect to $B_{i}$.

PROOF: In what follows, think of player 0 as having a singleton strategy space (fixed at $b_{0}$ ), so that $d m_{0}\left(b_{0}, \theta_{0}\right)=d P_{0}\left(\theta_{0}\right)$. Consider any Borel $E \subset \mathcal{B} \times \Theta$,

$$
\begin{aligned}
B(E) & =\int_{E}\left[\prod_{i=0}^{n} d m_{i}\left(b_{i} \mid \theta_{i}\right)\right] d P(\theta) \\
& =\int_{E} f(\theta)\left[\prod_{i=0}^{n} d m_{i}\left(b_{i} \mid \theta_{i}\right) d P_{i}\left(\theta_{i}\right)\right] \\
& =\int_{E} f(\theta) \prod_{i=0}^{n} d m_{i}\left(b_{i}, \theta_{i}\right) \\
& =\int_{E} f(\theta) d m .
\end{aligned}
$$


Given that $f(\theta)<M$ (Assumptions 2 and 3), it follows that $B(E)<M m(E)$. Since $m_{i}=B_{i},{ }^{46}$ we have established $B(E)<M \prod_{i} B_{i}(E)$ and hence part (i). Assume further that $0<M^{\prime}<f(\theta)$ (under Assumption 11) so that $B(E)>M^{\prime} m(E)$. This implies that $B(E)>M^{\prime} \prod_{i} B_{i}(E)$ establishing part (ii).

Q.E.D.

A useful implication of Lemma 17 is that information about the bids or values of some of the players only changes the probabilities of events involving other players by a factor of between $M$ and $M^{\prime}$.

LEMMA 18: Under Assumptions 2-4 and 11, if $J$ and $J^{\prime}$ are disjoint subsets of players, and $E_{J}$ is a positive probability event involving only the bids or values of players in $J$, and similarly for $E_{J^{\prime}}$, then

$$
M \operatorname{Pr}\left(E_{J}\right) \geq \operatorname{Pr}\left(E_{J} \mid E_{J^{\prime}}\right) \geq M^{\prime} \operatorname{Pr}\left(E_{J}\right) .
$$

PROOF: To see the second inequality (the first is analogous), note that

$$
\begin{aligned}
\operatorname{Pr}\left(E_{J} \mid E_{J^{\prime}}\right) & =\frac{\operatorname{Pr}\left(E_{J} \cap E_{J^{\prime}}\right)}{\operatorname{Pr}\left(E_{J^{\prime}}\right)} \\
& \geq M^{\prime} \frac{\operatorname{Pr}\left(E_{J}\right) \operatorname{Pr}\left(E_{J^{\prime}}\right)}{\operatorname{Pr}\left(E_{J^{\prime}}\right)} \\
& =M^{\prime} \operatorname{Pr}\left(E_{J}\right),
\end{aligned}
$$

where the inequality follows from the proof of Lemma 17.

Q.E.D.

Say that $b \in[\underline{b}, \bar{b}]$, is a bid-atom for $B_{i}$ if $B_{i}\left(\left\{b_{i h}=b\right.\right.$ for some $\left.\left.h\right\}\right)>0$. For each $i$, let $\widehat{B}_{i}$ be the set of bid-atoms of $B_{i}$.

Recall that our definition of a tie was constructed to rule out irrelevant cases in which a small change in bid does not affect the allocation. Say that $b_{i h}$ and $b_{j h^{\prime}}$ are in a pre-tie if $b_{i h}=b_{j h^{\prime}}$. Let $Y_{i} \equiv \bigcup_{j \neq i}\left(\widehat{B}_{j}\right)$. By avoiding $b_{i} \in Y_{i}, i$ avoids pre-ties with any given $j \neq i$, and so the probability that he is involved in a pre-tie (and hence a tie) is 0 . This is stated in the following lemma.

LEMMA 19: Under Assumptions 2-4, $\operatorname{Pr}\left(\left\{b_{i h}=b_{j h^{\prime}}\right\} \cap\left\{b_{i h} \notin Y_{i}\right\}\right)=0$. That is, there is zero probability of a pre-tie involving $i$ when $i$ does not use bids in $Y_{i}$.

Proof: This is obvious if the $B_{i}$ are independent. The result follows by absolute continuity of $B$ with respect to $\prod_{i=1}^{n} B_{i}$ (Lemma 17, part (i)).

Q.E.D.

ProOf OF Lemma 7: Choose a sequence $X=\left\{x_{1}, x_{2}, \ldots\right\}$ that is dense on $[\underline{b}, \bar{b}]$ but avoids $Y_{i}$. Since $Y_{i}$ is countable, this can be done. For any $\varepsilon>0$, define the mapping $\psi_{i}^{\varepsilon}: \Theta_{i} \times \mathcal{B}_{i} \rightarrow \Theta_{i} \times \mathcal{B}_{i}$ as follows.

If $v_{i h}>p_{\text {ihe }}\left(b_{i h}\right)$, let $\psi_{i}^{\varepsilon}\left(e_{i}, v_{i}, b_{i}\right)=\left(e_{i}, v_{i}, b_{i}^{\prime}\right)$, where $b_{i h}^{\prime}$ is the lowest indexed element of $X$ such that $b_{i h} \leq b_{i h}^{\prime} \leq b_{i h}+\varepsilon$, and such that $b_{i h}^{\prime}$ is below the next discretely higher bid than $b_{i h} \cdot{ }^{47}$ If

${ }^{46} B$ is defined by $d B(b, \theta)=\prod_{i} d m_{i}\left(b_{i} \mid \theta_{i}\right) d P(\theta)$. The careful reader can verify that the marginal of $B$ on $i, B_{i}$, has the same form as one would obtain by directly taking the marginal of $P$ onto $P_{i}$, which leads to $d m_{i}\left(b_{i} \mid \theta_{i}\right) d P_{i}\left(\theta_{i}\right)$ (which for a distributional strategy is the same as $\left.d m_{i}\left(b_{i}, \theta_{i}\right)\right)$.

${ }^{47}$ It is useful to note here that raising a buy bid indicates a greater desire to buy, while raising a sell bid indicates a lower willingness to sell. In each case, since $v_{i h}>p_{i h e_{i}}\left(b_{i h e_{i}}\right)$, this is the direction of the player's incentives if he is on the margin between being allocated or not allocated an object $h$. 
$v_{i h} \leq p_{\text {ihe }_{i}}\left(b_{i h}\right)$, let $\psi_{i}^{\varepsilon}\left(e_{i}, v_{i}, b_{i}\right)=\left(e_{i}, v_{i}, b_{i}^{\prime}\right)$, where $b_{i h}^{\prime}$ is the lowest indexed element of $X$ such that $b_{i h} \geq b_{i h}^{\prime} \geq b_{i h}-\varepsilon$, and such that $b_{i h}^{\prime}$ is above the next discretely lower bid than $b_{i h}$. Since $X$ is dense, such elements exist (and by Zorn's lemma $b_{i h}^{\prime}$ is unique).

So, $\psi_{i}^{\varepsilon}$ slightly raises bids where value is above the marginal transfer, and slightly lowers bids where value is at or below the marginal transfer in such a way as to miss elements of $Y_{i}$.

Note that the resulting bid vector is still an element of $\mathcal{B}_{i}$. To see this, note that for any $h^{\prime}>h$, if $b_{i h}>b_{i h^{\prime}}$ then by construction $b_{i h}>b_{i h}^{\prime}$. If $b_{i h}=b_{i h^{\prime}}$, then, since $p_{i h e_{i}}(x)$ is nondecreasing in $h$, and since $v_{i h}$ is nonincreasing, it cannot simultaneously be the case that $v_{i h} \leq p_{i h e_{i}}\left(b_{i h}\right)$ and $v_{i h^{\prime}}>p_{i h^{\prime} e_{i}}\left(b_{i h^{\prime}}\right)$. Hence, the algorithm will never specify lowering $b_{i h}$ but raising $b_{i h^{\prime}}$. And, if it specifies the same direction of movement, it will also specify the same outcome, so that again there will be no nonmonotonicity of the resultant bid vector.

Next, let us check that $\psi_{i}^{\varepsilon}$ is measurable. To see this, fix $i$ and $h$, and for each $j \in\{1,2,3, \ldots\}$, let $R_{j}$ be the set of $\left(e_{i}, v_{i}, b\right)$ such that either $v_{i h}>p_{i h e_{i}}\left(b_{i h}\right), b_{i h} \leq x_{j} \leq b_{i h}+\varepsilon$, and $x_{j}$ is below the next discretely different bid than $b_{i h}$ or $v_{i h} \leq p_{i h e_{i}}\left(b_{i h}\right), b_{i h} \geq x_{j} \geq b_{i h}-\varepsilon$, and $x_{j}$ is above the next discretely different bid than $b_{i h} . R_{j}$ is the finite union of the finite intersection of measurable sets, and so is measurable.

But, in fact, $\psi^{-1}\left(x_{j}\right)=R_{j} \backslash \bigcup_{j^{\prime}<j} R_{j}$, and so is itself measurable. As $X$ is countable, and as the image of $\psi$ is contained in $X$, the result follows. ${ }^{48}$

Hence, $m_{i} \circ \psi_{i}^{\varepsilon}$ is a well defined distributional strategy. By construction, under $m_{i} \circ \psi_{i}^{\varepsilon}$, player $i$ is involved in pre-ties with probability zero, and $m_{i} \circ \psi_{i}^{\varepsilon}$ can be made arbitrarily close to $m_{i}$.

The proof is completed by noting that when $m_{i} \circ \psi_{i}^{\varepsilon}$ buys or sells additional objects compared to $m_{i}$, it does so at prices that are at worst a little unfavorable. More formally, consider any $\left(e_{i}, v_{i}, b_{i}, e_{-i}, b_{-i}\right)$ and the $b^{\prime}$ generated by $\psi_{i}^{\varepsilon}$. Let $a_{i}$ be the allocation $i$ received with $b$ (given whatever randomizations the auctioneer performed), and $a_{i}^{\prime}$ be the allocation under $b^{\prime}$. Note that with probability one, $\left(e_{i}, v_{i}, b_{i}^{\prime}, e_{-i}, b_{-i}\right)$ is such that $i$ is not involved in any ties given $b^{\prime}$, so here randomizations are irrelevant. Now, if $a^{\prime}=a$, then $i$ 's payoffs have changed by at most $z \varepsilon$, where $z$ is the maximum slope of $t_{i}$ for any given endowment and allocation. Assume $a^{\prime}>a$. Then, for each $h \in\left(a+1, \ldots, a^{\prime}\right)$, it must be that $v_{i}>p_{i h e_{i}}\left(b_{i h}\right)$. This is actually not completely obvious, as one way in which the player might have picked up an extra object is that one of his other bids was relevant to whatever odd tie-breaking rule the auctioneer might have been using. But, in the event that $v_{i} \leq p_{i h e_{i}}\left(b_{i h}\right), b_{i h}^{\prime}$ is strictly lower than $b_{i h}$. So, since $i$ was not allocated an object $h$ before (implying that $b_{i h}$ was at best in a tie), then he certainly will not be allocated an object $h$ now. Note next that it must have been the case that each $b_{i h}, h \in\left(a+1, \ldots, a^{\prime}\right)$, was within $\varepsilon$ of being involved in a tie (since it was consistent not to allocate these objects to $i$ before, but it is consistent now). Hence, the increase in $i$ 's payment, given moving from allocation $a$ to $a^{\prime}$, is at most

$$
\begin{aligned}
\sum_{h \in\left(a+1, \ldots, a^{\prime}\right)}\left(p_{i h e_{i}}\left(b_{i h}\right)+2 z \varepsilon\right) & \leq 2 z \ell \varepsilon+\sum_{h \in\left(a+1, \ldots, a^{\prime}\right)} p_{i h e_{i}}\left(b_{i h}\right) \\
& \leq 2 z \ell \varepsilon+\sum_{h \in\left(a+1, \ldots, a^{\prime}\right)} v_{i h} .
\end{aligned}
$$

Hence, the difference between the increase in payments and the value of the extra objects allocated is at most $2 z \ell \varepsilon$.

Q.E.D.

ProOf OF LeMma 8: Fix an arbitrary player $i$. Let

$$
\widehat{o}_{i}(e, b, v)[h] \equiv \sum_{a_{i}=h}^{\ell} o^{\prime}(e, b, v)\left[a_{i}\right]
$$

${ }^{48}$ Note that we have only sorted out those $\left(e_{i}, v_{i}, b_{i}\right)$ such that $b_{i}^{\prime}$ has $h$ th element $x$. It is of course trivial to sort out which $\left(e_{i}, v_{i}, b_{i}\right)$ get mapped into any given $\ell$ vector from $X$. 
be the probability that $i$ ends up with $h$ or more objects given $(e, b, v)$. Let $T_{i}$ be the event that $i$ is involved in a tie given equilibrium play. Let $T_{i h}^{\text {buy }}$ be the subset of $T_{i}$ such that $b_{i h}$ is a buy offer involved in a tie and $\widehat{o}_{i}(e, v, b)[h]<1$. So, $T_{i h}^{\text {buy }}$ is the set of events where $i$ 's $h$ th bid is involved in a tie and $i$ does not get an $h$ th object for sure, given at least one of $b_{i h}>0, v_{i h}>0$. Suppose that $\operatorname{Pr}\left(T_{i h}^{\text {buy }}\right)>0$ under $m^{\prime}$. By Lemma $19, \operatorname{Pr}\left(b_{i h} \in Y_{i} \mid T_{i h}^{\text {buy }}\right)=1$. By Lemma $5, \operatorname{Pr}\left(v_{i h}<\right.$ $\left.p_{i h e_{i}}\left(b_{i h}\right) \mid T_{i h}^{\text {buy }}\right)=0$. By atomlessness (Assumption 4$), \operatorname{Pr}\left(v_{i h} \in p_{i h e_{i}}\left(Y_{i}\right) \mid T_{i h}^{\text {buy }}\right)=0$. It follows that $\operatorname{Pr}\left(v_{i h}>p_{i h e_{i}}\left(b_{i h}\right) \mid T_{i h}^{\text {buy }}\right)=1$.

Similarly, let $T_{i h}^{\text {sell }}$ be the subset of $T_{i}$ such that $i$ 's $h$ th bid is a sell offer involved in a tie and $\widehat{o}_{i}(e, b, v)[h]>0$. Recalling that in our set up, a sale occurs when one's bid is not accepted, this is the set of events where $i$ 's $h$ th bid is involved in a tie, and $i$ does not sell object $h$ for sure. By an argument analogous to that establishing that $\operatorname{Pr}\left(v_{i h}>p_{i h e_{i}}\left(b_{i h}\right) \mid T_{i h}^{\text {buy }}\right)=1$, it follows that $\operatorname{Pr}\left(v_{i h}<p_{i h e_{i}}\left(b_{i h}\right) \mid T_{i h}^{\text {sell }}\right)=1$.

Define

$$
\begin{aligned}
\omega \equiv & \sum_{\left\{h \mid \operatorname{Pr}\left(T_{i h}^{\text {sell }}\right)>0\right\}} \operatorname{Pr}\left(T_{i h}^{\text {sell }}\right) E\left(\widehat{o}_{i}(e, b, v)[h]\left(p_{i h e_{i}}\left(b_{i h}\right)-v_{i h}\right) \mid T_{i h}^{\text {sell }}\right) \\
& +\sum_{\left\{h \mid \operatorname{Pr}\left(T_{i h}^{\text {buy }}\right)>0\right\}} \operatorname{Pr}\left(T_{i h}^{\text {buy }}\right) E\left(\left(1-\widehat{o}_{i}(e, b, v)[h]\right)\left(v_{i h}-p_{i h e_{i}}\left(b_{i h}\right)\right) \mid T_{i h}^{\text {buy }}\right) .
\end{aligned}
$$

So, the first term sums the probabilities of not making a sale on unit $h$, multiplied by the minimum expected profit on that sale (under Assumption 7), and the second sum does the same thing for purchases. If $\operatorname{Pr}\left(T_{i h}^{\text {sell }}\right)$ or $\operatorname{Pr}\left(T_{i h}^{\text {buy }}\right)$ is positive for any $i, h$, then $\omega$ is positive.

Suppose $\omega>0$. Consider changing $m_{i}$ to $m \circ \psi_{i}^{\varepsilon}$, for $\varepsilon$ small. It gains at least $\omega$ by transforming $\widehat{o}_{i}(e, b, v)[h]$ to 0 (which, recall, means that $i$ sells his $h$ th object for sure) when a sell bid $b_{i h}$ would have been involved in a tie, and $\widehat{o}_{i}(e, b, v)[h]$ to 1 when a buy bid $b_{i h}$ would have been involved in a tie. And, as argued above, it does almost as well as $m_{i}$ in terms of the trading price on objects that would have traded anyway, and on any new trades created beyond those from converting what were originally ties. So, this deviation is strictly profitable for $\varepsilon$ sufficiently small. Q.E.D.

The proof of Lemma 10 uses the following lemma.

Let $c_{i}\left(e_{i}, v_{i}, b_{i}\right)$ be the distance between $\left(e_{i}, v_{i}, b_{i}\right)$ and $W_{i}$. Of course, $c_{i}$ is continuous. Define $C_{i}\left(m_{i}\right)$ as the expectation of $c_{i}(\cdot)$ given $m_{i}$.

LEMMA 20: Let $o$ and $m$ be arbitrary. For each $i$, and for each $\varepsilon>0$, there is a strategy $m_{i}^{*}$ such that $C\left(m_{i}^{*}\right)<\varepsilon$ and such that $\pi\left(m_{i}^{*}, m_{-i}, o\right) \geq \pi(m, o)-\varepsilon$.

PROOF: First replace $m_{i}$ by $m_{i}^{\prime}$ as given by Lemma 7 such that $m_{i}^{\prime}$ is tie-free for $i$, and such that $\pi_{i}\left(m_{i}^{\prime}, m_{-i}, o\right) \geq \pi_{i}\left(m_{i}, m_{-i}, o\right)-\varepsilon / 2$. Consider $\omega_{i} \circ m_{i}^{\prime}$ as given in Assumption 10 . Under $o_{S}$ (standard tie-breaking) this does weakly better, so that $\pi_{i}\left(\omega_{i} \circ m_{i}^{\prime}, m_{-i}, o_{S}\right) \geq$ $\pi_{i}\left(m_{i}^{\prime}, m_{-i}, o_{S}\right)$. And, of course, $C_{i}\left(m_{i}^{\prime}\right)=0$. Now, conceivably, $\omega_{i}\left(m_{i}^{\prime}\right)$ is not tie-free. Once again using Lemma 7, let $m_{i}^{*}$ be a tie-free strategy for $i$ close enough to $\omega_{i}\left(m_{i}^{\prime}\right)$, such that $\pi_{i}\left(m_{i}^{*}, m_{-i}, o_{S}\right) \geq \pi_{i}\left(\omega_{i}\left(m_{i}^{\prime}\right), m_{-i}, o_{S}\right)-\varepsilon / 2$, and such that $C_{i}\left(m_{i}^{*}\right) \leq \varepsilon$. Then,

$$
\begin{aligned}
\pi_{i}\left(m_{i}^{*}, m_{-i}, o\right) & =\pi_{i}\left(m_{i}^{*}, m_{-i}, o_{S}\right) \\
& \geq \pi_{i}\left(\omega_{i}\left(m_{i}^{\prime}\right), m_{-i}, o_{S}\right)-\varepsilon / 2 \\
& \geq \pi_{i}\left(m_{i}^{\prime}, m_{-i}, o_{S}\right)-\varepsilon / 2 \\
& =\pi_{i}\left(m_{i}^{\prime}, m_{-i}, o\right)-\varepsilon / 2 \\
& \geq \pi_{i}\left(m_{i}, m_{-i}, o\right)-\varepsilon .
\end{aligned}
$$


Proof: First, let us argue that $m$ must have support in $W_{i}$ for all $i$. Suppose not, so that for some bidder $i, m_{i}$ puts positive weight outside $W_{i}$. But, using Lemma 20, there is $m_{i}^{*}$ such that $C\left(m_{i}^{*}\right)<C\left(m_{i}\right) / 3$ and such that $\pi\left(m_{i}^{*}, m_{-i}, o\right) \geq \pi(m, o)-C\left(m_{i}\right) / 3$, and so $m_{i}^{*}$ does strictly better in $G$ than $m_{i}$.

Second, let us argue that $m$ is in fact an equilibrium of the original game. Assume not, so that for some $i$, there is $m_{i}^{\prime}$ such that $\pi_{i}\left(m_{i}^{\prime}, m_{-i}, o\right)>\varepsilon$, for some $\varepsilon>0$. Then, applying Lemma 20 once again, note that there is $m_{i}^{*}$ such that $C\left(m_{i}^{*}\right)<\varepsilon / 3$ and such that $\pi\left(m_{i}^{*}, m_{-i}, o\right) \geq$ $\pi(m, o)-\varepsilon / 3$. But then, $m_{i}^{*}$ also does strictly better in $G$ than $m_{i}$, a contradiction. Q.E.D.

ProOF OF COROLLARY 14: First, let us argue that there exists a monotone pure strategy equilibrium for a modified version of the auction. Modify our auction as follows. Add a small probability that the seller's (player 0 's) endowment takes on any value between 0 and $n \ell$, that he sets a zero reserve price, and that there is an additional bidder who enters the auction and randomly makes $2 n \ell$ bids with an atomless full support on admissible bids. Trivial extensions of Theorems 6 and 9 admit this modification. Thus, there exists an undominated* equilibrium of the modified game that does not involve any competitive ties and remains an equilibrium under any omniscient and effectively trade-maximizing tie-breaking rule.

Consider an arbitrary agent $i$, and let us argue that agent $i$ 's strategy must be monotone and pure.

We start with the following claim.

ClAIM 21: Let $v_{i}^{\prime}$ and $v_{i}$ be such that $v_{i h}^{\prime}>v_{i h}$ for all $h$, and $b_{i}^{\prime}$ be a best response for $v_{i}^{\prime}$, and $b_{i}$ be a best response for $v_{i}$. Then $b_{i}^{\prime} \geq b_{i}$.

PROOF: Suppose to the contrary that $b_{i h}^{\prime}<b_{i h}$ for at least one $h$. We will argue that this leads to a contradiction.

By Theorem 9, we know that the equilibrium is still an equilibrium if we choose a tie-breaking rule such that $i$ loses any competitive ties. So, let us assume that this is the tie-breaking rule.

Next, due to the added uncertainty of the aggregate endowment and the presence of the extra bidder, we know that any change of $b_{i h}$ leads to a change in the probability of winning an $h$ th object. Under the tie-breaking rule where $i$ loses all competitive ties, a change in $b_{i h}$, leaving $i$ 's other bids unchanged, does not change the probability that $i$ wins at least $h-1$ objects, or at least $h+1$ objects. Thus, under (Assumption $8^{\prime}$ ), we can write $i$ 's expected payoff given $\left(e_{i}, v_{i}, b_{i}\right)$ in the following way: ${ }^{49}$

$$
\left(\sum_{h} v_{i h} P_{i h}\left(b_{i h}\right)\right)-E\left[T_{i}(e, b) \mid e_{i}, b_{i}\right],
$$

where $P_{i h}\left(b_{i h}\right)$ is the probability of winning at least $h$ objects given $i$ 's $h$ th bid.

Choose a contiguous set of indexes $H=\left\{h_{L}, \ldots, h_{H}\right\}$ such that $b_{i h}^{\prime}<b_{i h}$ for $h \in H$, while $b_{i h}^{\prime} \geq b_{i h}$ for $h \in\left\{h_{L}-1, h_{H}+1\right\}$ the two bids immediately to either side of $H$ (except if $H$ contains either 0 or $\ell$ ). Then, note that $d_{i}^{\prime}$, defined by starting from $b_{i}^{\prime}$ and raising bids in $H$ to $b_{i h}$ is a valid bid vector, because $b_{i h_{L}-1}^{\prime} \geq b_{i h_{L}-1}$. Similarly, $d_{i}$, defined by starting from $b_{i}$ and lowering bids in $H$ to $b_{i h}^{\prime}$ is a valid bid vector, because $b_{i h_{H}+1}^{\prime} \geq b_{i h_{H}+1}$.

Since $b_{i}$ is a best response at $v_{i}$,

$$
\sum_{h} v_{i h} P_{i h}\left(b_{i h}\right)-E\left(T_{i}\left(e, b_{-i}, b_{i}\right)\right) \geq \sum_{h} v_{i h} P_{i h}\left(d_{i h}\right)-E\left(T_{i}\left(e, b_{-i}, d_{i}\right)\right) .
$$

${ }^{49}$ Note that $T_{i}$ may not be defined in situations where $i$ is involved in a competitive tie. However, given the tie-breaking rule such that $i$ always loses competitive ties and the continuity of the $t_{i}$ 's, we can easily extend $T_{i}$ to be defined at the (measure 0 ) set of points where $i$ is involved in a competitive tie by approximating with bids from "below." 
And, since only bids in $H$ have changed, and since the tie-breaking rule has $i$ always lose competitive ties, it follows that

$$
\sum_{h \in H} v_{i h}\left(P_{i h}\left(b_{i h}\right)-P_{i h}\left(d_{i h}\right)\right) \geq E\left(T_{i}\left(e, b_{-i}, b_{i}\right)-T_{i}\left(e, b_{-i}, d_{i}\right)\right)
$$

and so, since $d_{i h}=b_{i h}^{\prime}$ on $H$,

$$
\sum_{h \in H} v_{i h}\left(P_{i h}\left(b_{i h}\right)-P_{i h}\left(b_{i h}^{\prime}\right)\right) \geq E\left(T_{i}\left(e, b_{-i}, b_{i}\right)-T_{i}\left(e, b_{-i}, d_{i}\right)\right) .
$$

Similarly, since $b_{i}^{\prime}$ is a best response at $v_{i}^{\prime}$,

$$
\sum_{h} v_{i h}^{\prime} P_{i h}\left(b_{i h}^{\prime}\right)-E\left(T_{i}\left(e, b_{-i}, b_{i}^{\prime}\right)\right) \geq \sum_{h} v_{i h} P_{i h}\left(d_{i h}^{\prime}\right)-E\left(T_{i}\left(e, b_{-i}, d_{i}^{\prime}\right)\right)
$$

and so

$$
\sum_{h \in H} v_{i h}^{\prime}\left(P_{i h}\left(b_{i h}^{\prime}\right)-P_{i h}\left(d_{i h}^{\prime}\right)\right) \geq E\left(T_{i}\left(e, b_{-i}, b_{i}^{\prime}\right)-T_{i}\left(e, b_{-i}, d_{i}^{\prime}\right)\right),
$$

from which

$$
\sum_{h \in H} v_{i h}^{\prime}\left(P_{i h}\left(b_{i h}^{\prime}\right)-P_{i h}\left(b_{i h}\right)\right) \geq E\left(T_{i}\left(e, b_{-i}, b_{i}^{\prime}\right)-T_{i}\left(e, b_{-i}, d_{i}^{\prime}\right)\right) .
$$

Adding (3) to (2), one obtains

$$
\begin{aligned}
& \sum_{h \in H}\left(v_{i h}-v_{i h}^{\prime}\right)\left(P_{i h}\left(b_{i h}\right)-P_{i h}\left(b_{i h}^{\prime}\right)\right) \\
& \quad \geq E\left(T_{i}\left(e, b_{-i}, b_{i}\right)-T_{i}\left(e, b_{-i}, d_{i}\right)\right)-E\left(T_{i}\left(e, b_{-i}, d_{i}^{\prime}\right)-T_{i}\left(e, b_{-i}, b_{i}^{\prime}\right)\right) .
\end{aligned}
$$

Now, since $v_{i h}<v_{i h}^{\prime}$ for all $h$, and since $b_{i h}>b_{i h}^{\prime}$ for all $h \in H$, and since, given the perturbation, any change in bid sometimes matters, the left-hand side of this expression is strictly negative. However, by Assumption $8^{\prime}$, the right-hand side of this expression is $0 .{ }^{50}$ This is a contradiction, and so our supposition was incorrect.

Q.E.D.

Now let us complete the proof of the corollary. By Claim 21, along any line in agent $i$ 's valuation space that passes through one valuation vector and another vector that is strictly higher in each dimension, the best response correspondence is increasing in the strong sense that the smallest best response at $v^{\prime}>v$ is at least as large as the largest best response at $v$. Thus, along any such line, there are at most a countable number of points at which the best response correspondence is multi-valued. This implies that the set of valuation vectors where the best response correspondence is multi-valued is of $\ell$-dimensional Lebesgue measure 0 . To see this, denote the set of such valuation vectors by $A$. By Fubini's Theorem, write the $\ell$-dimensional Lebesgue measure of $A$ as

$$
\int \cdots \int I_{A}\left(v_{i 1}, v_{i 2}, \ldots, v_{i \ell}\right) d v_{i 1} \cdots d v_{i \ell},
$$

${ }^{50}$ Note that while Assumption $8^{\prime}$ only applies to a change in a single bid at a time, we can write the right-hand side of (2) as a sum of changes of a single bid at a time iterating over the bids in $H$ (here from highest indexed to lowest), and similarly we can write the right-hand side of (3) as a sum of changes that involve only a single bid in $H$ at a time (here from lowest indexed to highest). Each $h$ th bid where $h \in H$, changes exactly once in the corresponding sum in both cases, and so we can then apply Assumption 8'. 
where $I_{A}$ is the indicator function. Without loss of generality for assessing the measure of $A$, we can assume that it is any line where we vary only $v_{i 1}$ that intersects $A$ in at most a countable number of points. ${ }^{51}$ This implies that

$$
\int I_{A}\left(v_{i 1}, v_{i 2}, \ldots, v_{i \ell}\right) d v_{i 1}=0
$$

for all $\left(\cdot, v_{i 2}, \ldots, v_{i \ell}\right)$. It follows that

$$
\int \cdots \int I_{A}\left(v_{i 1}, v_{i 2}, \ldots, v_{i \ell}\right) d v_{i 1} \cdots d v_{i \ell}=0 .
$$

Since the distribution of types is absolutely continuous with respect to $\ell$-dimensional Lebesgue measure, this implies that the measure of types for which $i$ 's best response is multi-valued is 0 . Hence, by Claim 21, it follows that the equilibrium must be in monotone pure strategies. ${ }^{52}$

Finally, let us now take the limit as the extra noise vanishes (along a subsequence if necessary). By Theorem 2 in JSSZ, we obtain a monotone pure strategy undominated* equilibrium of the limit game; but possibly with a strange tie-breaking rule. ${ }^{53}$ However, from Theorem 9 we know that the equilibrium must not involve any competitive ties and remains an equilibrium under any omniscient and effectively trade-maximizing tie-breaking rule, including standard tiebreaking.

Q.E.D.

Proof of Theorem 15: Fix an auction $\mathcal{A}=\left(P, o, t, b_{0}\right)$ satisfying Assumptions 1-13. For $x \in$ $\{3,4, \ldots\}$, consider an auction $\mathcal{A}^{x}$ modified from $\mathcal{A}$ as follows: With probability $1 / x$ a nonstrategic player $n+1$ has $e_{n+1}=1$ and submits a sell offer which is uniform on $[\underline{w}, \bar{w}]$. With probability $1 / x, e_{n+1}=0$ and $n+1$ submits a buy offer which is uniform on $[\underline{w}, \bar{w}]$. With residual probability, player $n+1$ is not involved. These events are independent of the events under $P$.

It is a trivial extension of Theorem 6 that each $A^{x}$ has an undominated* equilibrium $m^{x}$. We show that there is $\rho>0$ independent of $x$ such that $\operatorname{Pr}\left(\left\{\right.\right.$ trade under $\left.\left.m^{x}\right\}\right)>\rho$ for all $x$. By JSSZ Theorem 2 (which shows upper hemi-continuity of augmented equilibrium strategies and allocations as the parameters of the game change), any accumulation point of $\left\{m^{x}\right\}$ is an (augmented) equilibrium of $\mathcal{A}$ that puts probability one on the closure of the set of undominated strategies for which the probability of trade is at least $\rho$. By Theorem 6 , this is a standard equilibrium, and so we are done. (A proof of this step via Reny is also possible.)

So, assume that there is no such $\rho$. Then, there exists a subsequence of $x$ such that the probability of trade under the corresponding $m^{x}$ goes to 0 along the subsequence. With a renaming, we can assume that trade goes to 0 along the original sequence.

For notational convenience, let $\operatorname{Pr}_{x}(E)$ be the probability of event $E$ happening in auction $x$ under $m^{x}$. When the probability of an event does not depend on $x$, we write simply $\operatorname{Pr}(E)$.

Consider the case that condition (i) of Assumption 12 is satisfied. (The argument for condition (ii) is analogous.) Let $H$ be the set of buyers for whom the max of the support over buy values is $\bar{w}$. Then, by atomlessness, there is $\omega>0$ such that for each $i \in H$, there is a probability

${ }^{51}$ For the purpose of determining whether $A$ has positive Lebesgue measure, we can simply rotate the set itself-so that lines that passed through it at " 45 degrees" are now vertical in the $v_{i 1}$ dimension. We omit a change of notation where $A$ is replaced by its rotation $A^{\prime}$. Alternatively, one can do a change of variables, and reach the same conclusion.

${ }^{52}$ Given that we are dealing with distributional strategies, this is up to sets of measure 0 , which is in accordance with our definition of monotone pure strategies. One can change the strategies on the sets of measure 0 , if one likes.

${ }^{53}$ Note that the set of pure monotone strategies is compact, and that when one takes limits of strategies and outcomes, the limit tie-breaking rule may differ from those on the sequence, but will be an omniscient one. 
of at least $\omega$ that there are at least $\ell+1$ sell values below $\bar{w}-\omega$ among $N \backslash i$, and such that for each $i \notin H, i$ never has a buy value above $\bar{w}-\omega$.

Consider an arbitrary $k \in\{3,4, \ldots\}$, and let $\delta<\omega / k$. For $i=1, \ldots, n+1$, let $Q_{B i}^{x}$ be the number of buy bids above $\bar{w}-2 \delta$ that $i$ makes. So,

$$
\left\{Q_{B i}^{x}>0\right\}=\left\{e_{i}<\ell, b_{i, e_{i}+1}>\bar{w}-2 \delta\right\} .
$$

For each $x$, let

$$
\mu_{x}=\max _{i \in H} \operatorname{Pr}_{x}\left\{Q_{B i}^{x}>0\right\}
$$

be the maximum probability that any player (other than $n+1$ ) makes a buy bid above $\bar{w}-2 \delta$ in $\mathcal{A}^{x}$ (by Assumption 13, the fact that $m^{x}$ is undominated ${ }^{*}$, and choice of $\omega, \operatorname{Pr}_{x}\left(Q_{B i}^{x}>0\right)=0$ for each player in $N \backslash H)$. Let $i^{x}$ be an associated maximizer of $\mu_{x}$.

Let $Q_{B}^{x}=\sum_{i=1}^{n+1} Q_{B i}^{x}$ be the random variable giving the number of buy bids above $\bar{w}-2 \delta$ under $m^{x}$ by any player (including player $n+1$ ). Let $Q_{S}^{x}$ be the number of sell bids at or below $\bar{w}-2 \delta$ by anyone other than $i^{x}$ (but including player $n+1$ ).

Let us first argue that $\operatorname{Pr}_{x}\left(Q_{B}^{x}>0\right) \rightarrow 0$ must hold. To see this, suppose to the contrary that everywhere along a subsequence, $\operatorname{Pr}_{x}\left(Q_{B}^{x}>0\right)>\gamma$, for some $\gamma>0$. Let $j, j^{\prime}$ be any two players who sometimes have a sell value below $\bar{w}-\omega$ (two such players exist, since there are sometimes at least $\ell+1$ sell values below $\bar{w}-\omega)$. Then, along a subsequence, at least one of the players, say player $j$, assesses probability at least $\gamma / 2$ that one of his opponents makes a buy bid above $\bar{w}-2 \delta$ for each $x$. By Lemma 18, $j$ thus assigns probability at least $M^{\prime} \gamma / 2$ of such a bid conditional on $j$ having a sell value below $\bar{w}-\omega$. Consider the strategy for $j$ that whenever he has a sell value below $\bar{w}-\omega$, he bids $\bar{w}-2 \delta$ on that unit, and that he sets all other bids equal to value (thus guaranteeing a nonnegative profit on those units). Let $\tau_{j}$ be the probability of such a value for $j$.

Consider first the case where $j$ wins at least half the time when he bids $\bar{w}-2 \delta$ and one of his opponents makes a buy bid above $\bar{w}-2 \delta$. Then, by A13, his utility from this strategy is at least

$$
\frac{\tau_{j}}{2} U(\omega-2 \delta) M^{\prime} \gamma / 2 .
$$

But, as the utility of selling is bounded by $U(\bar{w})-U(\underline{w})$ (since $\bar{w}$ is the most one will ever receive, and the value of the unit sold is at least $\underline{w}$ ), this implies that in equilibrium, $j$ is selling with probability bounded away from zero, contradicting that trade goes to zero along the sequence.

Consider next the case where $j$ wins less than half the time when he bids $\bar{w}-2 \delta$ and one of his opponents makes a buy bid above $\bar{w}-2 \delta$. This can only happen if at least half the time that this occurs, there is another player making a sell bid below $\bar{w}-2 \delta$. But in this event, even under $j$ 's equilibrium strategy, at least one unit will have been transferred. Hence, trade is occurring at least

$$
\tau_{j} M^{\prime} \gamma / 2
$$

of the time, which again contradicts the fact that the probability of trade is going to zero.

So, we have established that $\operatorname{Pr}_{x}\left(Q_{B}^{x}>0\right) \rightarrow 0 . \operatorname{Pr}_{x}\left(Q_{S}^{x}>0\right) \rightarrow 0$ must also hold. Assume not, so that $\operatorname{Pr}_{x}\left(Q_{S}^{x}>0\right)>\gamma>0$ along a subsequence. Choose a subsequence along which $i^{x}$ is constant. Let $Y_{i^{x}}$ be the event that $i^{x}$ has a buy value above $\bar{w}-\delta$. Since $i^{x} \in H, \operatorname{Pr}\left(Y_{i^{x}}\right)>0$. Now, since $Q_{S}^{x}$ depends only on players other than $i^{x}, \operatorname{Pr}_{x}\left(Q_{S}^{x}>0 \mid Y_{i^{x}}\right)>M^{\prime} \gamma$ (once again using Lemma 18). So, consider the deviation for $i^{x}$ in which he bids $\bar{w}-2 \delta$ whenever $Y_{i^{x}}$ holds, and bids his value otherwise (which under Assumption 13 can never result in a loss). Then, we can argue as before: either $i^{x}$ wins at least half the time that $Q_{S}^{x}>0$ and $Y_{i^{x}}$ are true, in which case his surplus (and hence equilibrium probability of winning) are bounded away from 0 , or $i^{x}$ wins less than half the time, in which case the equilibrium amount of trade is bounded positive. Either is a contradiction. 
Let

$$
\hat{\mu}_{x}=\max \left(\mu_{x}, \frac{1}{x} \frac{2 \delta}{\bar{w}-\underline{w}}\right)>0
$$

be the maximum over probabilities that any player (including player $n+1$ ) makes a buy offer greater than $\bar{w}-2 \delta$.

Note that $\operatorname{Pr}_{x}\left(Q_{B}^{x}>0\right) \geq \hat{\mu}_{x}$ and hence $\hat{\mu}_{x}$ must go to 0 . Note also that

$$
\operatorname{Pr}_{x}\left(Q_{B}^{x}>0\right) \leq \sum_{i=1}^{n+1} \operatorname{Pr}_{x}\left(Q_{B i}^{x}>0\right) \leq n \hat{\mu}_{x},
$$

since the second expression errs only in that it overcounts situations where $\operatorname{Pr}_{x}\left(Q_{B i}^{x}>0\right)$ for more than one $i$.

The probability that $q \geq 2$ players bid at or above $\bar{w}-2 \delta$ is less than $(n ! /(q !(n-q) !)) M^{q-1}\left(\hat{\mu}_{x}\right)^{q}$, where the factor $M^{q-1}$ takes account of the fact that once one knows that one (or more) players have bid at or above $\bar{w}-2 \delta$, the likelihood of further such players may increase to $M \mu_{x}$. It follows that

$$
\operatorname{Pr}_{x}\left\{Q_{B}^{x}>\ell\right\}<\phi\left(\left(\hat{\mu}_{x}\right)^{2}+\left(\hat{\mu}_{x}\right)^{3}+\cdots+\left(\hat{\mu}_{x}\right)^{n}\right),
$$

where $\phi<\infty$ accounts for both the combinatorial terms and a factor of $M^{n-1}$. Since $\operatorname{Pr}_{x}\left\{Q_{B}^{x}>\right.$ $0\} \geq \hat{\mu}_{x}$, and since $\hat{\mu}_{x} \rightarrow 0$, it follows from the above inequality that

$$
\operatorname{Pr}_{x}\left(Q_{B}^{x} \leq \ell \mid Q_{B}^{x}>0\right) \rightarrow 1 .
$$

Choose a subsequence along which $i^{x}$ is constant. Let $\zeta$ be the probability that $i^{x}$ has a buy value above $\bar{w}-2 \delta$. Since $i^{x} \in H, \zeta>0$. Consider the deviation $d_{j}$ for any given $j \neq i^{x}$ that whenever $j$ has a sell value $v_{j h}$ below $\bar{w}-\omega$, and the equilibrium specifies a bid $b_{j h}$ above $\bar{w}-2 \delta$, $j$ submits $b_{j h}=\bar{w}-2 \delta$ instead. We show that this is improving for at least one $j \neq i^{x}$.

Consider the following set of events, measured relative to the equilibrium strategies. The $E$ 's should be indexed by $x$, but we omit the notation.

$E_{1}^{\prime}: Q_{B i^{x}}^{x}>0$.

$E_{1}^{\prime \prime}: Q_{B, n+1}^{x}>0, i^{x}$ has a buy value above $\bar{w}-2 \delta$.

$E_{2}: Q_{S}^{x}=0$.

$E_{3 j}$ : Player $j$ has an unsold unit with value below $\bar{w}-\omega$, even though player $j$ sells strictly less than $Q_{B}^{x}$ objects.

$E_{4}: Q_{B}^{x} \leq \ell$.

$E_{5}$ : There are at least $\ell+1$ sell values below $\bar{w}-\omega$ among $N \backslash i^{x}$.

If $\hat{\mu}_{x}$ is maximized by $i_{x}$, let $E_{1}=E_{1}^{\prime}$. If $\hat{\mu}_{x}$ is maximized by $n+1$, let $E_{1}=E_{1}^{\prime \prime}$. Then, in the first case, $\operatorname{Pr}_{x}\left(E_{1}\right)=\hat{\mu}_{x}$, while in the second, $\operatorname{Pr}_{x}\left(E_{1}\right)=\operatorname{Pr}\left(Q_{B, n+1}>0\right) \operatorname{Pr}\left(i^{x}\right.$ has a buy value above $\bar{w}-2 \delta) \geq \zeta \hat{\mu}_{x}$, since player $n+1$ is independent of the rest of the system. In either case, $\operatorname{Pr}_{x}\left(E_{1}\right) \geq \zeta \hat{\mu}_{x}$.

Note that under either $E_{1}^{\prime}$ or $E_{1}^{\prime \prime}, i^{x}$ has a buy value above $\bar{w}-2 \delta$, and hence, since marginal utility is decreasing and $m^{x}$ is undominated*, Assumption 13 implies that $i^{x}$ has no sell bids at or below $\bar{w}-\delta$. Under $E_{2}$, no player other than $i^{x}$ has a sell bid at or below $\bar{w}-2 \delta$ either. Thus, under $E_{1} \cap E_{2}$, there is no sell bid of $\bar{w}-2 \delta$ or lower. So, under $E_{1} \cap E_{2} \cap E_{3 j}, j$ sells at least one extra object by $d_{j} . E_{4}$ and $E_{5}$ are not necessary for $j$ to sell an extra object. Their role will become clear momentarily.

We claim that $\operatorname{Pr}_{x}\left(E_{2} \mid E_{1}\right) \rightarrow 1$. To see this in the case of $E_{1}=E_{1}^{\prime}$, recall that we have argued that $\operatorname{Pr}_{x}\left(E_{2}\right) \rightarrow 1$, and hence $\operatorname{Pr}_{x}\left(\neg E_{2}\right) \rightarrow 0$. But $\operatorname{Pr}_{x}\left(\neg E_{2} \mid E_{1}\right) \leq M \operatorname{Pr}_{x}\left(\neg E_{2}\right)$ since $E_{2}$ involves only players other than $i^{x}$, while $E_{1}^{\prime}$ only involves player $i^{x}$. Hence $\operatorname{Pr}_{x}\left(\neg E_{2} \mid E_{1}\right) \rightarrow 0$, and so $\operatorname{Pr}_{x}\left(E_{2} \mid E_{1}\right) \rightarrow 1$. In the case of $E_{1}^{\prime \prime}$, there is also the information that player $n+1$ bid at or above $\bar{w}-2 \delta$. This implies that $n+1$ did not make any sell bid. Since $n+1$ is independent of the rest of the players, the claim again follows. 
Next, note that $\operatorname{Pr}_{x}\left(E_{5} \mid E_{1}\right) \geq M^{\prime} \omega$, since $\operatorname{Pr}_{x}\left(E_{5}\right) \geq \omega$, and since $E_{1}$ involves only players $i^{x}$ and $n+1$, while $E_{5}$ involves neither of these players.

Finally, $\operatorname{Pr}_{x}\left(E_{4} \mid E_{1}\right) \rightarrow 1$ by (6). Combining these, it follows that

$$
\operatorname{Pr}_{x}\left(E_{2} \cap E_{4} \cap E_{5} \mid E_{1}\right) \geq \frac{M^{\prime} \omega}{2}
$$

for $x$ sufficiently large (draw a Venn diagram), and hence

$$
\operatorname{Pr}\left(E_{1} \cap E_{2} \cap E_{4} \cap E_{5}\right) \geq \frac{M^{\prime} \omega}{2} \zeta \hat{\mu}_{x} .
$$

Thus, $d_{j}$ earns at least $\omega-2 \delta$ with probability at least

$$
\begin{aligned}
\operatorname{Pr}_{x}\left(E_{1} \cap E_{2} \cap E_{3 j}\right) & \geq \operatorname{Pr}_{x}\left(E_{1} \cap E_{2} \cap E_{3 j} \cap E_{4} \cap E_{5}\right) \\
& \geq \operatorname{Pr}_{x}\left(E_{3 j} \mid E_{1} \cap E_{2} \cap E_{4} \cap E_{5}\right) \frac{M^{\prime} \omega}{2} \zeta \hat{\mu}_{x} .
\end{aligned}
$$

On the other hand, lowering the sell bid on object $h$ to $\bar{w}-2 \delta$ can only result in a worse outcome for $j$ than the equilibrium strategy when $j$ would already have sold object $h$, which occurs with probability bounded by $\operatorname{Pr}_{x}\left(Q_{B}^{x}>0\right)$. From $5, \operatorname{Pr}_{x}\left(Q_{B}^{x}>0\right) \leq n \hat{\mu}_{x}$. And, since $\bar{w}$ was the upper bound on buy values for players, and hence on their bids, these objects would have sold for at most $\bar{w}$, while under $d_{j}$, they sell for at least $\bar{w}-2 \delta$. So, the cost of $d_{j}$ in this event is at most $\ell 2 \delta$. Since the utility function has a bounded derivative, there exists $\Lambda<\infty$ such that $U^{\prime}(x) / U^{\prime}(y)<\Lambda$ for all $x$ and $y$. So, for the deviation not to be profitable, it must be that

$$
\operatorname{Pr}_{x}\left(E_{3 j} \mid E_{1} \cap E_{2} \cap E_{4} \cap E_{5}\right) \frac{M^{\prime} \omega}{2} \zeta \hat{\mu}_{x}(\omega-2 \delta) \leq n \hat{\mu}_{x} \ell 2 \delta \Lambda .
$$

Dividing both sides by $\hat{\mu}_{x} \delta$ (which is valid, because $\hat{\mu}_{x}$ is positive thanks to player $n+1$ ), and summing across players other than $i^{x}$,

$$
\frac{M^{\prime} \omega}{2} \zeta\left(\frac{\omega}{\delta}-2\right) \sum_{j \neq i^{x}} \operatorname{Pr}_{x}\left(E_{3 j} \mid E_{1} \cap E_{2} \cap E_{4} \cap E_{5}\right) \leq(n-1) n \ell 2 \Lambda .
$$

But, in any realization where $E_{1} \cap E_{2} \cap E_{4} \cap E_{5}$ holds, $E_{3 j}$ must hold for at least one $j \neq i^{x}$, since there are $\ell+1$ sell values below $\bar{w}-2 \delta$, since no sell bid is at or below $\bar{w}-2 \delta$, and since there are at most $\ell$ buy offers above $\bar{w}-2 \delta$ (it is for this purpose that $E_{4}$ and $E_{5}$ were maintained). Integrating across realizations,

$$
\sum_{j \neq i^{x}} \operatorname{Pr}_{x}\left(E_{3 j} \mid E_{1} \cap E_{2} \cap E_{4} \cap E_{5}\right) \geq 1,
$$

and so

$$
\frac{M^{\prime} \omega}{2} \zeta\left(\frac{\omega}{\delta}-2\right) \leq(n-1) n 2 \ell \delta \Lambda .
$$

Recall that $k \in\{3,4, \ldots\}$ is arbitrary and that $\delta$ was chosen so that $\omega / k>\delta$. It follows that

$$
\frac{M^{\prime} \omega}{2} \zeta(k-2) \leq(n-1) n 2 \ell \frac{\omega}{k} \Lambda .
$$

This equation is clearly false for $k$ sufficiently large, and we have a contradiction. 


\section{REFERENCES}

Athey, S. (2001): "Single Crossing Properties and the Existence of Pure Strategy Equilibria in Games of Incomplete Information," Econometrica, 69, 861-890.

BAJARI, P. (1996): "Properties of the First Price Sealed Bid Auction with Asymmetric Players," Mimeo, Stanford University.

Blume, A. (2003): "Bertrand Without Fudge," Economics Letters, 78, 167-168.

Bresky, M. (1999): "Equilibrium in Multi-Unit Auctions,” Mimeo, CERGE, Prague.

CRAmTON, P. C. (1995): "Money Out of Thin Air: The Nationwide Narrowband PCS Auction," Journal of Economics and Management Strategy, 4, 267-343.

CRIPPS, M., AND J. M. Swinkels (2003): "Depth and Efficiency of Large Private Value Double Auctions," Mimeo, Washington University in St. Louis.

DASGUPTA, P., AND E. MASKIN (1986): "The Existence of Equilibrium in Discontinuous Economic Games I: Theory," Review of Economic Studies, 53, 1-26.

Dubey, P., AND M. ShubiK (1978): "A Theory of Money and Financial Institutions, 28: The Noncooperative Equilibria of a Closed Trading Economy with Market Supply and Bidding Strategies," Journal of Economic Theory, 17, 1-20.

FudenberG, D., M. MoBIUS, AND A. SzeIDL (2003): "Large Auctions,” Mimeo, Harvard University.

JACKSON, M. O. (1999): “The Non-Existence of Equilibrium in Auctions with Two Dimensional Types," Mimeo, California Institute of Technology.

JACKSON, M. O., AND I. KREMER (2004): "The Relevance of a Choice of Auction Format in a Competitive Environment," California Institute of Technology, Social Science Working Paper 1190.

JaCkson, M. O., L. K. Simon, J. M. Swinkels, AND W. R. ZAME (2002): "Communication and Equilibrium in Discontinuous Games of Incomplete Information," Econometrica, 70, 1711-1740.

JACKSON, M. O., AND J. M. Swinkels (1999): "Existence of Equilibrium in Auctions and Discontinuous Bayesian Games: Endogenous and Incentive Compatible Sharing Rules," Mimeo, California Institute of Technology.

KadAn, O. (2004): "Equilibrium in the Two Player $k$-Double Auction with Affiliated Private Values," Mimeo, Washington University in St. Louis.

KAZUMORI, E. (2003): "Toward a Theory of Strategic Markets with Incomplete Information: Existence of Isotone Equilibria," Mimeo, California Institute of Technology.

KRISHNA, V. (2003): "Asymmetric English Auctions," Journal of Economic Theory, 112, 261-288.

LizZERI, A., AND N. PERSICO (2000): "Uniqueness and Existence of Equilibrium in Auctions with a Reserve Price," Games and Economic Behavior, 30, 83-114.

LEBRUN, B. (1996): "Existence of an Equilibrium in First-Price Auctions," Economic Theory, 7, 421-443.

(1999): "First Price Auctions in the Asymmetric $N$ Player Case," International Economic Review, 40, 125-142.

MANELl, A. (1996): "Cheap Talk and Sequential Equilibria in Signaling Game," Econometrica, 64, 917-942.

MAskin, E., AND J. Riley (2000): "Equilibrium in Sealed High Bid Auctions," Review of Economic Studies, 67, 439-452.

MCADAMS, D. (2003): “Isotone Equilibria in Games of Incomplete Information," Econometrica, $71,1191-1214$.

Milgrom, P., AND R. J. WeBer (1982): "A Theory of Auctions and Competitive Bidding," Econometrica, 50, 1089-1122.

(1985): "Distributional Strategies for Games with Incomplete Information," Mathematics of Operations Research, 10, 619-632.

PeCK, J. D., K. Shell, AND S. SPEAR (1992): "The Market Game: Structure and Existence of Equilibrium,” Journal of Mathematical Economics, 21, 271-299. 
PERRY, M., AND P. J. RENY (2003): “Toward a Strategic Foundation for Rational Expectations Equilibrium,” Working Paper, University of Chicago.

RENY, P. J. (1999): "On the Existence of Pure and Mixed Strategy Nash Equilibria in Discontinuous Games,” Econometrica, 67, 1029-1056.

RENY, P. J., AND S. ZAMIR (2003): "On the Existence of Pure Strategy Monotone Equilibria in Asymmetric First-Price Auctions," Econometrica, 72, 1105-1126.

SimON, L. K. (1987): "Games with Discontinuous Payoffs," Review of Economic Studies, 54, 569-597.

SimON, L. K., AND W. R. ZAME (1990): "Discontinuous Games and Endogenous Sharing Rules," Econometrica, 58, 861-872.

(1999): "Cheap Talk and Discontinuous Games of Incomplete Information,” Working Paper, University of California at Los Angeles.

SwinKElS, J. M. (1999): "Asymptotic Efficiency for Discriminatory Private Value Auctions," Review of Economic Studies, 66, 509-528. (2001): "Efficiency of Large Private Value Auctions," Econometrica, 69, 37-68.

VICKREY, W. (1961): “Counterspeculation, Auctions, and Competitive Sealed-Tenders," Journal of Finance, 16, 8-37.

WiLliams, S. (1991): "Existence and Convergence of Equilibria in the Buyer's Bid Double Auction," Review of Economic Studies, 58, 351-374. 